\title{
Flavoured leptogenesis and $\mathrm{CP}^{\mu \tau}$ symmetry
}

\author{
Rome Samanta ${ }^{a}$ and Manibrata Sen ${ }^{b, c}$ \\ ${ }^{a}$ Physics and Astronomy, University of Southampton, \\ Southampton, SO17 1BJ, U.K. \\ ${ }^{b}$ Department of Physics, University of California Berkeley, \\ Berkeley, CA 94720, U.S.A. \\ ${ }^{c}$ Department of Physics and Astronomy, Northwestern University, \\ Evanston, IL 60208, U.S.A. \\ E-mail: R.Samanta@soton.ac.uk, manibrata@berkeley.edu
}

ABSTRACT: We present a systematic study of leptogenesis in neutrino mass models with $\mu \tau$-flavoured CP symmetry. In addition to the strong hierarchical $N_{1}$-dominated scenario $\left(N_{1} \mathrm{DS}\right)$ in the 'two flavour regime' of leptogenesis, we show that one may choose the right-handed $(\mathrm{RH})$ neutrino mass hierarchy as mild as $M_{2} \simeq 4.7 M_{1}$ for a perfectly valid hierarchical $N_{1}$ DS. This reduces the lower bound on the allowed values of $M_{1}$, compared to what is stated in the literature. The consideration of flavour effects due to the heavy neutrinos also translate into an upper bound on $M_{1}$. It is only below this bound that the observed baryon-to-photon ratio can be realized for a standard $N_{1}$ domination, else a substantial part of the parameter space is also compatible with $N_{2}$ DS. We deduce conditions under which the baryon asymmetry produced by the second $\mathrm{RH}$ neutrino plays an important role. Finally, we discuss another scenario where lepton asymmetry generated by $N_{2}$ in the two flavour regime faces washout by $N_{1}$ in the three flavour regime. Considering a hierarchical light neutrino mass spectrum, which is now favoured by cosmological observations, we show that at the end of $N_{1}$-leptogenesis, the asymmetry generated by $N_{2}$ survives only in the electron flavour and about $33 \%$ of the parameter space is consistent with a pure $N_{2}$-leptogenesis.

Keywords: Cosmology of Theories beyond the SM, CP violation, Neutrino Physics

ARXIV EPRINT: 1908.08126 


\section{Contents}

1 Introduction 1

$\begin{array}{lll}2 & \mathrm{CP}^{\mu \tau} & \text { symmetry and its variants in seesaw model }\end{array}$

3 Validity of $\mathrm{N}_{1} \mathrm{DS}$ in one flavour thermal leptogenesis 5

4 Flavour effects and importance of $N_{2}$-leptogenesis $\quad 11$

5 Leptogenesis in the $\mathrm{CP}^{\boldsymbol{\mu} \tau}$ symmetric model $\mathbf{1 5}$

5.1 Two flavour regime: $10^{9} \mathrm{GeV}<M_{1,2}<10^{12} \mathrm{GeV} \quad 18$

$5.210^{9} \mathrm{GeV}<M_{2}<10^{12} \mathrm{GeV}$ and $M_{1}<10^{9} \mathrm{GeV} 23$

$\begin{array}{llr}6 & \text { Summary } & 26\end{array}$

\section{Introduction}

Neutrino masses and mixings continue to intrigue. Precise measurement of the six mixing parameters - the three mixing angles: solar $\left(\theta_{12}\right)$, atmospheric $\left(\theta_{23}\right)$ and reactor $\left(\theta_{13}\right)$, the two mass-squared differences: solar $\left(\Delta m_{12}^{2}\right)$, and atmospheric $\left(\Delta m_{23}^{2}\right)$, and the CP phase $\delta$ - is essential for a clear understanding of neutrino physics. While we have almost zeroed in on the values of the mixing angles and mass-squared differences from solar, atmospheric and terrestrial experiments [1], we are still pretty much in the dark when it comes to the $\mathrm{CP}$ phase. To this end, significant improvements have been made to the experimental determination of $\delta$ in experiments such as $\mathrm{T} 2 \mathrm{~K}[2-4]$ and $\mathrm{NO} \nu \mathrm{A}[5,6]$. There exists a mild preference for the normal mass ordering (NMO) of the neutrinos, while latest global fit of neutrino oscillation data [7] seem to favour the second octant of $\theta_{23}$ (the best-fit value $\sin ^{2} \theta_{23}=0.58$ ), and a maximal value of the CP phase $\delta=3 \pi / 2$ (driven by T2K neutrino and anti-neutrino appearance data) for both the mass orderings. However, precise statements on the mass ordering, octant of $\theta_{23}$ and the value of $\delta$ are yet to be made with a high degree of confidence level.

This is an exciting time in low energy neutrino phenomenology. Models which have concrete predictions for the yet undetermined parameters such as $\theta_{23}$ and $\delta$ can be tested in the light of recent experimental data. From a theory standpoint, flavour symmetries [8-11] have always been invoked in neutrino mass models to estimate neutrino mixing parameters. A popular example is the $\mu \tau$ symmetry [12-19], which was ruled out by the discovery of a non-zero $\theta_{13}$. However, after the hint of maximal $\mathrm{CP}$ violation by $\mathrm{T} 2 \mathrm{~K}[2]$, another variant of the $\mu \tau$ symmetry, the $\mu \tau$ flavoured $\mathrm{CP}$ symmetry $\left(\mathrm{CP}^{\mu \tau}\right)$ or $\mu \tau$ reflection symmetry [20-22] has been a topic of interest in the recent years [23-42]. 
The $\mathrm{CP}^{\mu \tau}$ symmetry, which is a CP transformation [43-45] on the left-handed (LH) neutrino fields with $\mu \tau$ interchange symmetry as the CP generator in the low energy effective neutrino Lagrangian, predicts a co-bimaximal mixing [46]: $\theta_{23}=\pi / 4$ and $\delta=$ $\pi / 2,3 \pi / 2$, along with arbitrary non-zero values of $\theta_{13}$. To make $\mathrm{CP}^{\mu \tau}$ more predictive, a sizable body of work exists to combine flavour symmetries with CP symmetries, despite this being a non-trivial task $[25,26]$. Several aspects of $\mathrm{CP}^{\mu \tau}$ and its alternative versions have also been explored [47-56].

From the point of view of cosmology, the $\mathrm{CP}^{\mu \tau}$ model has generated considerable interest in the possibility of baryogenesis via leptogenesis [58-61, 63, 64]. In an extended Standard Model (SM), augmented with right-handed ( $\mathrm{RH})$ neutrinos, tiny masses for the active neutrinos can be generated through the Type-I seesaw mechanism [65-67]. In such models, CP-violating and out of equilibrium decays of the heavy $\mathrm{RH}$ neutrinos can generate a lepton asymmetry (leptogenesis) which can be converted into a baryon asymmetry (baryogenesis) by sphalerons [57, 58,60]. These sphaleronic transitions conserve B - L, and violate $\mathrm{B}+\mathrm{L}$, where $\mathrm{B}$ and $\mathrm{L}$ are the baryon and lepton number respectively. Given a neutrino mass model, successful baryogenesis requires [68]

$$
\eta_{B}^{t h} \equiv \eta_{B}^{C M B}=(6.3 \pm 0.3) \times 10^{-10},
$$

where $\eta_{B}^{t h}$ and $\eta_{B}^{C M B}$ are the theoretical and observed values of baryon to photon ratio at the recombination. Assuming a $N_{1}$ dominated scenario $\left(N_{1} \mathrm{DS}\right)$, where only the decays and interactions of $N_{1}$ matter, it has been pointed out that $\mathrm{CP}^{\mu \tau}[22,69,70]$ as well as the $\mathrm{CP}$ symmetries similar to $\mathrm{CP}^{\mu \tau}$, e.g., $\mathrm{CP}$-anti $\mu \tau\left(\mathrm{CP}^{\mu \tau A}\right)$ [35], and complex scaling [52, 55] are capable of reproducing the observed value of $\eta_{B}$. This, however, requires the lightest RH neutrino mass to lie within the range $10^{9} \mathrm{GeV}<M_{1}<10^{12} \mathrm{GeV}$ - so called the two flavour regime (2FR) [71-74] of leptogenesis. For $\mathrm{CP}^{\mu \tau}$ as well as $\mathrm{CP}^{\mu \tau A}$, it has also been argued that the regimes $M_{1}>10^{12} \mathrm{GeV}$ - one flavour regime (1FR) and $M_{1}<10^{9} \mathrm{GeV}$ - three flavour regime (3FR) - are not favoured for successful leptogenesis due to the typical structure of the symmetry (we shall discuss it in detail in section 5 ). In the $N_{1} \mathrm{DS}$, leptogenesis has been studied with a strong hierarchical scenario [22, 35, 69], e.g., $M_{2} / M_{1}=10^{3}$; i.e., assuming other heavy neutrinos are not produced at all, or if produced, the lepton asymmetry due to $N_{2}$ faces a significant washout by the $N_{1}$-interactions and thus is negligible, whereas that produced by $N_{1}$ does not encounter a $N_{2}$-washout. In addition, a lower bound on $M_{1}$ has been derived $[22,35]$ using the neutrino oscillation data and the observed range of $\eta_{B}$.

In this paper we investigate viability of those results in detail. After a systematic analysis, we argue the following:

(i) In the $\mathrm{CP}^{\mu \tau}$ framework, even in the two $\mathrm{RH}$ neutrino seesaw model $[75,76]$ which is tightly constrained by the neutrino oscillation data, one can choose the heavy RH neutrino mass hierarchy as low as $M_{2} / M_{1} \simeq 4.7$ for a perfectly valid hierarchical $\mathrm{N}_{1}$ DS leptogenesis scenario. This in turn leads to a decrease in the lower bound on $M_{1}$, approximately by an order of magnitude. 
(ii) Allowing both the $\mathrm{RH}$ neutrinos to contribute to the final asymmetry and taking into account the heavy neutrino flavour effects, we show that in the two flavour regime, there is a particular RH neutrino mass window $M^{\max }>M_{1}>M^{\text {min }}$ for which the hierarchical $\mathrm{N}_{1}$ DS is valid. Beyond $M^{\mathrm{max}}$, domination of $N_{2}$ could also become significant in addition to $N_{1}$.

(iii) Finally, we demonstrate that if the lepton asymmetry is produced by $N_{2}$ in the two flavour regime and faces washout by $N_{1}$ in the three flavour regime, then the final asymmetry mainly survives in the electron flavour. This is because the $N_{1}$-decay parameters for the other two flavours $\left(K_{1 \mu}\right.$ and $\left.K_{1 \tau}\right)$ are strong enough to erase any pre-existing asymmetry in the respective flavours. We quantify the probability of $\mathrm{N}_{2}$ leptogenesis to be around $33 \%$. This is done by computing the probability of the electron flavour washout parameter $K_{1 e}$ to be less than unity, since typically for these values of $K_{1 e}$, the asymmetry generated by $N_{2}$ does not get washed out by $N_{1}[77-80] .^{1}$

The rest of the paper is organized as follows: in section 2 we briefly discuss the $\mathrm{CP}^{\mu \tau}$ and its other variants. For simplicity we only focus on the two RH neutrino model, commonly known as the minimal seesaw. Section 3 contains a discussion about the validity of $\mathrm{N}_{1}$ DS in one flavour case which can trivially be generalized into multi flavoured leptogenesis scenario. In section 4 , we emphasize on the importance of heavy neutrino flavour effects which open up the possibility for $N_{2}$ leptogenesis. Section 5 contains a thorough discussion of leptogenesis in the model under consideration. We conclude our work in section 6 emphasizing the main results of this work.

\section{$2 \mathrm{CP}^{\mu \tau}$ symmetry and its variants in seesaw model}

Before we proceed, we discuss some aspects of the $\mathrm{CP}^{\mu \tau}$ symmetry in neutrino mass models. Note that we work in a basis where the charged lepton mass matrix $m_{\ell}$ and the $\mathrm{RH}$ neutrino mass matrix $M_{R}$ are diagonal [22,69]. Thus, the neutrino mixing matrix $U$ can be written as

$$
U=P_{\phi} U_{P M N S} \equiv P_{\phi}\left(\begin{array}{ccc}
c_{12} c_{13} & e^{i \frac{\alpha}{2}} s_{12} c_{13} & s_{13} e^{-i\left(\delta-\frac{\beta}{2}\right)} \\
-s_{12} c_{23}-c_{12} s_{23} s_{13} e^{i \delta} & e^{i \frac{\alpha}{2}}\left(c_{12} c_{23}-s_{12} s_{13} s_{23} e^{i \delta}\right) & c_{13} s_{23} e^{i \frac{\beta}{2}} \\
s_{12} s_{23}-c_{12} s_{13} c_{23} e^{i \delta} & e^{i \frac{\alpha}{2}}\left(-c_{12} s_{23}-s_{12} s_{13} c_{23} e^{i \delta}\right) & c_{13} c_{23} e^{i \frac{\beta}{2}}
\end{array}\right),
$$

where $P_{\phi}=\operatorname{diag}\left(e^{i \phi_{1}}, e^{i \phi_{2}} e^{i \phi_{3}}\right)$ is an unphysical diagonal phase matrix and $c_{i j} \equiv \cos \theta_{i j}$, $s_{i j} \equiv \sin \theta_{i j}$ with the mixing angles $\theta_{i j}=[0, \pi / 2]$. CP violation enters in eq. (2.1) through the Dirac phase $\delta$ and the Majorana phases $\alpha$ and $\beta$. For simplicity, we focus on the two RH neutrino model [75, 76], commonly known as minimal seesaw model [81-84]. Thus with $m_{D}$ as the Dirac mass matrix, the neutrino part of the Lagrangian can be written as

$$
-\mathcal{L}_{\text {mass }}^{\nu, N}=\bar{N}_{R i}\left(m_{D}\right)_{i \alpha} \nu_{L \alpha}+\frac{1}{2} \bar{N}_{R i}\left(M_{R}\right)_{i j} \delta_{i j} N_{R j}^{C}+\text { h.c. },
$$

\footnotetext{
${ }^{1}$ Following [63] we address $K_{1 \alpha}$ as decay parameters throughout.
} 
with $l_{L \alpha}=\left(\nu_{L \alpha} e_{L \alpha}\right)^{T}$ as the SM lepton doublet of flavor $\alpha$ and $M_{R}=\operatorname{diag}\left(M_{1}, M_{2}\right)$, $M_{1,2}>0$. The effective light neutrino mass matrix is given by the standard seesaw relation

$$
M_{\nu}=-m_{D}^{T} M_{R}^{-1} m_{D}
$$

Now a CP transformation $[43,44]$ on the LH neutrino field, $\nu_{L l} \rightarrow i G_{l m} \gamma^{0} \nu_{L m}^{C}$, leads to the following invariance of the effective light neutrino mass matrix $M_{\nu}$ :

$$
G^{T} M_{\nu} G=M_{\nu}^{*}
$$

where $G$ is the generator matrix. If $G$ follows a $\mu \tau$-interchange symmetry [20, 21], i.e.,

$$
G=\left(\begin{array}{lll}
1 & 0 & 0 \\
0 & 0 & 1 \\
0 & 1 & 0
\end{array}\right)
$$

then the symmetry transformation in eq. (2.4) is known as a $\mu \tau$ flavoured CP transformation or $\mathrm{CP}^{\mu \tau}$ [22]. A simple alteration of $\mathrm{CP}^{\mu \tau}$ has recently been studied by one of the authors, by adding a minus sign to the right hand side of eq. (2.4). ${ }^{2}$ This symmetry, named as the $\mathrm{CP}$ anti- $\mu \tau$ or $\mathrm{CP}^{\mu \tau A}$ [35], could be recast as a symmetry transformation equation similar to eq. (2.4) as

$$
\mathcal{G}^{T} M_{\nu} \mathcal{G}=M_{\nu}^{*}
$$

with $\mathcal{G}=i G$. Intriguingly, the $\mu \tau$ symmetry $(G)$ and the $\mu \tau$ antisymmetry $(\mathcal{G})$ have completely different predictions when they are used as an ordinary field transformation, i.e., $\nu_{L l} \rightarrow G_{l m} \nu_{L m}$ or $\nu_{L l} \rightarrow \mathcal{G}_{l m} \nu_{L m}$ [85]. However in their CP-transformed versions, along with the diagonalization condition $U^{T} M_{\nu} U=M_{d}$, where $M_{d}=\operatorname{diag}\left(m_{1}, m_{2}, m_{3}\right)$, both the symmetries (eq. (2.4) and eq. (2.6)) lead to the same predictions [22, 35]

$$
\cos \delta=\sin \alpha=\sin \beta=0, \quad \theta_{23}=\pi / 4 .
$$

This is easy to understand. Consider a mass matrix $M_{\nu}$, which follows eq. (2.6). This can be written in the form

$$
M_{\nu}^{C P^{\mu \tau A}}=\left(\begin{array}{ccc}
i A & B & -B^{*} \\
B & C & i D \\
-B^{*} & i D & -C^{*}
\end{array}\right),
$$

where $A, D$ are real and $B, C$ are complex mass dimensional quantities which are a priori unknown. Now mass matrix in eq. (2.8) also satisfies the equation

$$
G^{T}\left(i M_{\nu}^{C P^{\mu \tau A}}\right) G=\left(i M_{\nu}^{C P^{\mu \tau A}}\right)^{*},
$$

which is basically a $\mathrm{CP}^{\mu \tau}$ transformation (eq. (2.4)). Thus, if a mass matrix follows $\mathrm{CP}^{\mu \tau A}$ invariance, ' $i$ ' times the same matrix also obeys $\mathrm{CP}^{\mu \tau}$ symmetry, and hence both the symmetries lead to similar phenomenological consequences. Henceforth, without lack of

\footnotetext{
${ }^{2}$ Note that the high energy symmetry could be very different than $\mathrm{CP}^{\mu \tau}$, as pointed out in [35].
} 
generality, we shall consider the CP-antisymmetric parametrization of $m_{D}$ as well as $M_{\nu}$ derived in [35].

For a diagonal $M_{R}$, eq. (2.6) is satisfied through the symmetry transformation on $m_{D}$ as $^{3}$

$$
m_{D} \mathcal{G}=-i m_{D}^{*}
$$

The most general form of $m_{D}$ that satisfies (2.10) can be parametrized as

$$
m_{D}=\left(\begin{array}{lll}
\sqrt{2} a_{1} e^{i \pi / 4} & b_{1} e^{i \theta_{1}} & i b_{1} e^{-i \theta_{1}} \\
\sqrt{2} a_{2} e^{i \pi / 4} & b_{2} e^{i \theta_{2}} & i b_{2} e^{-i \theta_{2}}
\end{array}\right)
$$

where the parameters $a_{1,2}, b_{1,2}$ and $\theta_{1,2}$ are real. Now using eq. (2.3), the effective light neutrino mass matrix $M_{\nu}$ can be written as

$$
\begin{aligned}
& M_{\nu}^{C P^{\mu \tau A}}= \\
& \left(\begin{array}{ccc}
-2 i\left(x_{1}^{2}+x_{2}^{2}\right) & -\sqrt{2} e^{i \pi / 4}\left(x_{1} y_{1} e^{i \theta_{1}}+x_{2} y_{2} e^{i \theta_{2}}\right) & -i \sqrt{2} e^{i \pi / 4}\left(x_{1} y_{1} e^{-i \theta_{1}}+x_{2} y_{2} e^{-i \theta_{2}}\right) \\
-\sqrt{2} e^{i \pi / 4}\left(x_{1} y_{1} e^{i \theta_{1}}+x_{2} y_{2} e^{i \theta_{2}}\right) & -\left(e^{2 i \theta_{1}} y_{1}^{2}+e^{2 i \theta_{2}} y_{2}^{2}\right) & -i\left(y_{1}^{2}+y_{2}^{2}\right) \\
-i \sqrt{2} e^{i \pi / 4}\left(x_{1} y_{1} e^{-i \theta_{1}}+x_{2} y_{2} e^{-i \theta_{2}}\right) & -i\left(y_{1}^{2}+y_{2}^{2}\right) & e^{-2 i \theta_{1}} y_{1}^{2}+e^{-2 i \theta_{2}} y_{2}^{2}
\end{array}\right) .
\end{aligned}
$$

In (2.12), new real parameters $x_{1,2}$ and $y_{1,2}$ are defined by scaling $a_{1,2}$ and $b_{1,2}$ with the square roots of the respective $\mathrm{RH}$ neutrino masses $M_{1,2}$, i.e.

$$
\frac{a_{1,2}}{\sqrt{M_{1,2}}}=x_{1,2}, \quad \frac{b_{1,2}}{\sqrt{M_{1,2}}}=y_{1,2} .
$$

A few comments on the matrix $M_{\nu}^{C P^{\mu \tau A}}$ are in order. Since $\operatorname{det}\left(M_{\nu}^{C P^{\mu \tau A}}\right)=0$, the lightest neutrino mass (either $m_{1}$ for a normal mass ordering or $m_{3}$ for an inverted mass ordering) has to vanish. Furthermore, one of the phases in $M_{\nu}^{C P^{\mu \tau A}}$ (say $\theta_{1}$ ) could be rotated with the phase matrix $P_{\phi}=\operatorname{diag}\left(1, e^{i \phi}, e^{-i \phi}\right)$ by the choice $\theta_{1}=-\phi$. Therefore, we are left only with the phase difference $\theta_{2}-\theta_{1}$, which can be renamed as $\theta$. Without loss of generality, this is equivalent to the choice $\theta_{1}=0$ and $\theta_{2}=\theta$ in $m_{D}$. For phenomenological analysis, we use this redefined phase $\theta$ for both $M_{\nu}^{C P^{\mu \tau A}}$ as well as $m_{D}$.

\section{Validity of $\mathrm{N}_{1} \mathrm{DS}$ in one flavour thermal leptogenesis}

In this section, we start by discussing the standard $N_{1}$ dominated leptogenesis $\left(\mathrm{N}_{1} \mathrm{DS}\right)$ scenario in the presence of another heavy neutrino $N_{2}$, assuming both of them are thermally produced [86-89] so that the reheating temperature $T_{\mathrm{RH}}>M_{1,2}$. To begin with, we focus on the one-flavour scenario (i.e., no charged lepton flavour effects). The overall conclusions drawn from one flavour approximation can easily be generalized in the presence

\footnotetext{
${ }^{3}$ We shall refer the reader refs. $[22,55,69]$ to have a look to realize how in the diagonal basis of $m_{\ell}$ and $M_{R}$, CP symmetry could be applied in the neutrino mass terms.
} 
of flavour effects, as we discuss later. The set of classical kinetic equations [63] relevant for leptogenesis could be written as

$$
\begin{aligned}
\frac{d N_{N_{i}}}{d z} & =-D_{i}\left(N_{N_{i}}-N_{N_{i}}^{\mathrm{eq}}\right), \quad \text { with } i=1,2, \\
\frac{d N_{B-L}}{d z} & =-\sum_{i=1}^{2} \varepsilon_{i} D_{i}\left(N_{N_{i}}-N_{N_{i}}^{\mathrm{eq}}\right)-\sum_{i=1}^{2} W_{i} N_{B-L},
\end{aligned}
$$

with $z=M_{1} / T$. The $N_{i}$ 's and $N_{B-L}$ are the abundances per $N_{1}$ 's in ultra relativistic thermal equilibrium. The equilibrium abundances of $N_{i}$ 's are given by $N_{i}^{\mathrm{eq}}=\frac{1}{2} z_{i}^{2} \mathcal{K}_{2}\left(z_{i}\right)$, where $\mathcal{K}_{2}\left(z_{i}\right)$ are the modified Bessel functions. The total CP asymmetry is quantified by $\varepsilon_{i}=\sum_{\alpha} \varepsilon_{i \alpha}$ where

$$
\varepsilon_{i \alpha}=\frac{\Gamma_{i \alpha}-\bar{\Gamma}_{i \alpha}}{\Gamma_{i}+\bar{\Gamma}_{i}}
$$

The flavoured $\mathrm{CP}$ asymmetry parameter $\varepsilon_{i \alpha}$ can be estimated as

$$
\begin{aligned}
\varepsilon_{i \alpha}= & \frac{1}{4 \pi v^{2} h_{i i}} \sum_{j \neq i} \operatorname{Im}\left\{h_{i j}\left(m_{D}\right)_{i \alpha}\left(m_{D}^{*}\right)_{j \alpha}\right\}\left[f\left(x_{i j}\right)+\frac{\sqrt{x_{i j}}\left(1-x_{i j}\right)}{\left(1-x_{i j}\right)^{2}+h_{j j}^{2}\left(16 \pi^{2} v^{4}\right)^{-1}}\right] \\
& +\frac{1}{4 \pi v^{2} h_{i i}} \sum_{j \neq i} \frac{\left(1-x_{i j}\right) \operatorname{Im}\left\{h_{j i}\left(m_{D}\right)_{i \alpha}\left(m_{D}^{*}\right)_{j \alpha}\right\}}{\left(1-x_{i j}\right)^{2}+h_{j j}^{2}\left(16 \pi^{2} v^{4}\right)^{-1}}
\end{aligned}
$$

where $h_{i j} \equiv\left(m_{D} m_{D}^{\dagger}\right)_{i j},\left\langle\phi^{0}\right\rangle=v / \sqrt{2}, x_{i j}=M_{j}^{2} / M_{i}^{2}$ and $f\left(x_{i j}\right)$ has the standard expression [35]. The decay parameter is given by

$$
K_{i} \equiv \frac{\Gamma_{D, i}(T=0)}{H\left(T=M_{i}\right)},
$$

where $H\left(T=M_{i}\right)$ is the Hubble paramter defined at the temperature $\left.T=M_{i}\right)$. Using $z_{i}=z \sqrt{x_{1 i}}$, the decay terms can be written as

$$
D_{i}=\frac{\Gamma_{D, i}}{H z}=K_{i} x_{1 i} z\left\langle 1 / \gamma_{i}\right\rangle,
$$

where the total decay rates $\Gamma_{D, i}=\bar{\Gamma}_{i}+\Gamma_{i}=\Gamma_{D, i}(T=0)\left\langle 1 / \gamma_{i}\right\rangle$ with $\left\langle 1 / \gamma_{i}\right\rangle$ 's as the thermally averaged dilution factors given by the ratios of two modified Bessel functions

$$
\left\langle 1 / \gamma_{i}\right\rangle=\frac{\mathcal{K}_{1}\left(z_{i}\right)}{\mathcal{K}_{2}\left(z_{i}\right)} .
$$

The washout factor $W_{i}$ typically contains three terms: the inverse decay term $W_{i}^{\mathrm{ID}}$, the $\Delta L=1$ scattering term $W_{i}^{\Delta L=1}$, and the nonresonant part of the $\Delta L=2 \operatorname{term} W_{i}^{\Delta L=2}$. For a strong washout scenario ${ }^{4}$ and hierarchical light neutrino masses, the scattering terms and the $\Delta L=2$ terms can be safely neglected [64,90,91]. Thus, the relevant washout term $W_{i} \simeq W_{i}^{\mathrm{ID}}$ can be written as (after properly subtracting the real intermediate state contribution of $\Delta L=2$ process [61])

$$
W_{i}^{\mathrm{ID}}=\frac{1}{4} K_{i} \sqrt{x_{1 i}} \mathcal{K}_{1}\left(z_{i}\right) z_{i}^{3} .
$$

\footnotetext{
${ }^{4}$ We show later that a strong wash-out scenario is preferred in the model under consideration.
} 
The final $B-L$ asymmetry could be written as

$$
N_{B-L}^{f}=N_{B-L}^{\mathrm{in}} e^{-\sum_{i} \int d z^{\prime} W_{i}\left(z^{\prime}\right)}+N_{B-L}^{\text {lepto }},
$$

where $N_{B-L}^{\text {in }}$ could be a possible pre-existing asymmetry $[92,93]$ at an initial temperature $T_{\text {in }}$. However in this work, we do not consider any possible pre-existing asymmetry which would impose additional constraints ${ }^{5}$ on the model parameter space [86, 94]. In fact, as we shall discuss, given the RH neutrino masses in our model, $10^{9} \mathrm{GeV}<M_{1}, M_{2}<$ $10^{12} \mathrm{GeV}-2 \mathrm{FR}$, it is not possible to washout a pre-existing asymmetry which is orthogonal to the direction of $N_{1}$-washout [83, 95]. Thus, the scenario of a pure leptogenesis from RH neutrino decay breaks down. Assuming standard thermal history of the universe, the final baryon-to-photon ratio can be written as

$$
\eta_{B}=a_{\text {sph }} \frac{N_{B-L}^{\text {lepto }}}{N_{\gamma}^{\text {rec }}} \simeq 0.96 \times 10^{-2} N_{B-L}^{\text {lepto }},
$$

where $N_{\gamma}^{\text {rec }}$ is the normalised photon density at the recombination and the sphaleron conversion coefficient $a_{\mathrm{sph}} \sim 1 / 3$. This theoretically calculated value of $\eta_{B}$ has to be compared with measured value given in eq. (1.1).

Before discussing validity of the $N_{1}$ DS in presence of $N_{i(i \neq 1)}$, let us introduce another important parameter $\delta_{1 i}=\left(M_{i}-M_{1}\right) / M_{1}$ which accounts for the mass difference between $M_{i}$ and $M_{1}$. This is related to $x_{1 i}$ as

$$
\sqrt{x_{1 i}}=1+\delta_{1 i} \Rightarrow z_{i}=z\left(1+\delta_{1 i}\right) .
$$

Armed with all the necessary prerequisites, we solve eqs. (3.1), and (3.2) for $N_{1}$ in the presence of washouts due to both $N_{1}$ and $N_{2}$. Note that for the fixed values of $z$ and $K_{2}$, the strength of the $N_{2}$-washout $\left(W_{2}^{\mathrm{ID}}\right)$ depends on $\delta_{21}$ (cf. eq. (3.8)). As a result, solutions of eq. (3.2) for different values of $\delta_{12}$ indicates a minimum, below which the effect of $W_{2}^{\mathrm{ID}}$ starts to become prominent. This helps to reproduce the standard hierarchical $N_{1}$ dominated scenario.

In figure 1 , we show the variation of the produced asymmetry, $\left|N_{B-L}\right|$, with $z$. In each figure, $N_{B-L}$ lines in red and blue correspond to the asymmetry produced by $N_{1}$ subjected to $N_{1}$, and $N_{1}+N_{2}$ washout respectively. The asymmetry showed in black is that produced by $N_{2}$ subjected to $N_{1}+N_{2}$ washout. Figures in the top and bottom panel are for $K_{1}=K_{2}=25$ and $K_{1}=K_{2}=5$ respectively.

It is clear from the top-left panel that for $\delta_{12}=1$, even if one takes into account the $N_{2}$ washout alongwith the $N_{1}$ washout, the final asymmetry perfectly coincide with standard $N_{1}$ DS. This is simply because the $N_{2}$ washout goes out of equilibrium before the asymmetry production due to $N_{1}$ stops. Thus, the final dynamics is governed by the inverse decays of $N_{1}$ (i.e., $N_{1}$ washout). On the other hand, the asymmetry produced

\footnotetext{
${ }^{5}$ By "pre-existing asymmetry" we mean asymmetries that may originate not only from heavier RH neutrinos but also from other external sources. These asymmetries may be large in magnitude. Therefore one needs several conditions on the flavoured decay parameters to washout the pre-existing asymmetry. In literature, somtimes these conditions are referred to as "strong thermal conditions". See e.g., refs. [96, 97].
} 

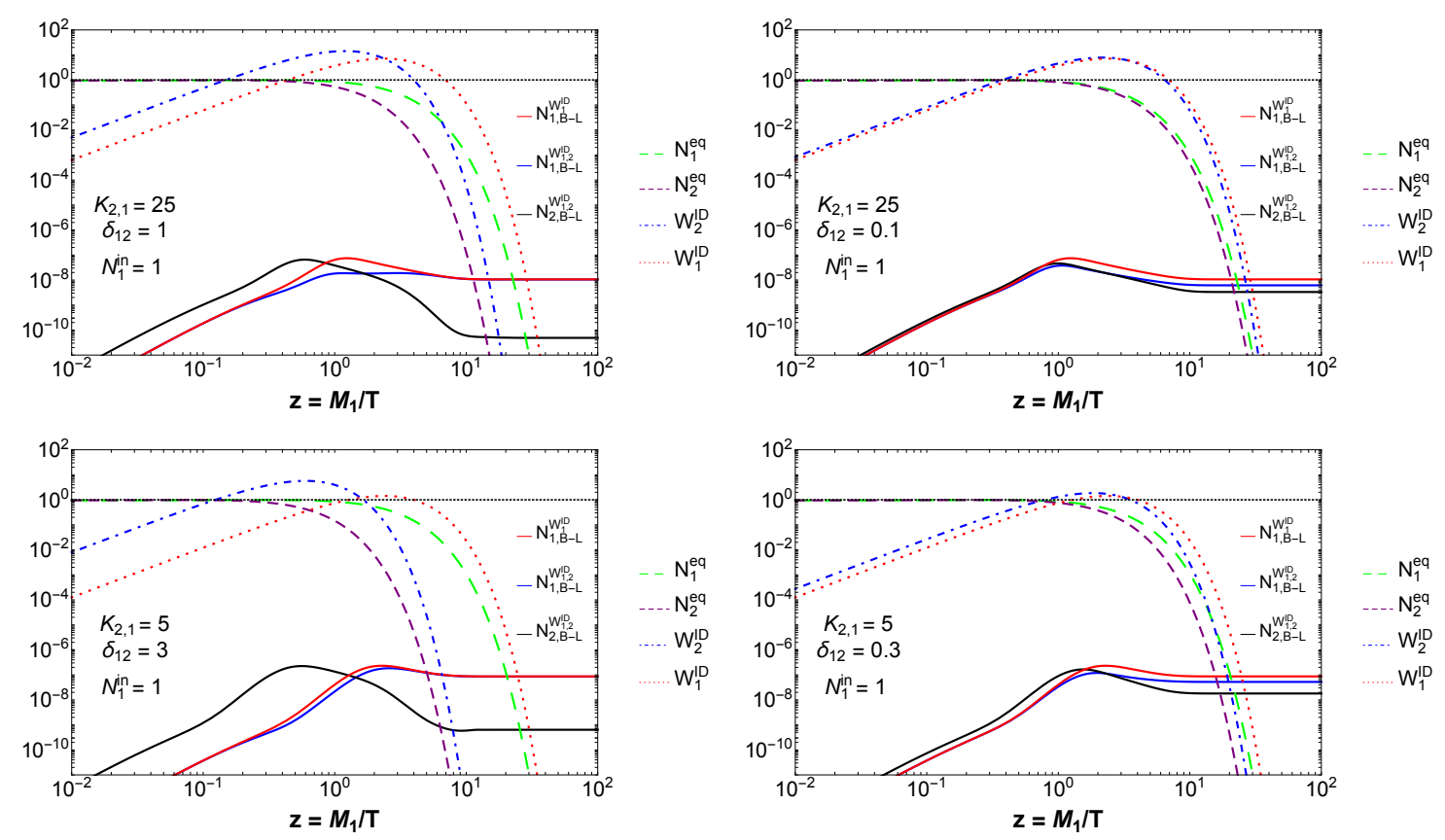

Figure 1. Top left: $\left|N_{B-L}\right|$ as a function of $z=M_{1} / T$ for the decay parameters $K_{1}=K_{2}=25$ (the other relevant quantities, e.g., $N_{1}^{\text {eq }}, W_{1}^{\mathrm{ID}}$ etc., are mentioned on the right side of each figure). Solid red line shows $\left|N_{B-L}\right|$ for a pure $N_{1}$ dominated scenario. The solid blue line shows the asymmetry generated by $N_{1}$, for $\delta_{12}=1$, subjected to both $N_{1}$ and $N_{2}$ washout, given by $W_{1,2}^{\text {ID }}$ respectively. The solid black line shows $N_{B-L}$, generated by $N_{2}$ subjected to $W_{1,2}^{\mathrm{ID}}$ washout. Top right: for the same value of the decay parameters we generate similar plots for $\delta_{12}=0.1$. Bottom panel shows similar plots as those in the top panel for $\delta_{12}=3$ (left) and $\delta_{12}=0.3$ (right), for $K_{1}=K_{2}=5$.

by $N_{2}$ is significantly washed out by $N_{1}$ (showed in black). This is due to the fact that when the strength of the $N_{1}$ inverse decay reaches its maximum value, the asymmetry production due to $N_{2}$ is practically switched off. On the top-right panel, we show the same quantities, but for $\delta_{12}=0.1$. Note that in this case, there is a clear distinction between a pure $N_{1}$ dominated scenario, and that where $N_{2}$ washout is also taken into account. Here, the $N_{2}$ washout of the asymmetry production due to $N_{1}$ cannot be ignored, and hence, the magnitude of $N_{B-L}$ reduces. Furthermore, the $N_{1}$ inverse decay cannot fully washout the asymmetry produced by $N_{2}$, since even when the $N_{1}$ washout is significant, asymmetry production due to $N_{2}$ does not cease. This causes a significant increase in the magnitude of the asymmetry produced by $N_{2}$. The bottom panel shows the same plots for $K_{1}=K_{2}=5$. In this case, however, pure $N_{1} \mathrm{DS}$ is realised with slightly increased value of $\delta_{12}=3$, as opposed to $\delta_{12}=1$. For completeness, we also show the plots with $\delta_{12}=0.3$ for which one cannot assume a pure $N_{1}$ DS due to the crucial role played by $N_{2}$.

This begs the following question: what is the minimum hierarchy in the $\mathrm{RH}$ neutrino masses so that a pure hierarchical $N_{1}$ DS is realized? For example, as discussed, if some model predicts a simple correlation between the decay parameters, say, $K_{1}=K_{2} \in(5-25)$, one can safely assume $\sqrt{x_{12}}=M_{2} / M_{1}=\left(1+\delta_{12}\right)=4$, so that the effect of $N_{2}$ washout at $N_{1}$-leptogenesis phase, as well as the asymmetry produced by $N_{2}$, can be neglected. 
However, for a realistic scenario, the correlation of the decay parameters may not be this simple; also, a realistic model might contain lots of data points constrained by neutrino oscillation data. Thus in terms of computation, it would be tedious to solve Boltzmann equations for each and every pair of decay parameters. It is useful then to consider explicit and accurate analytic formalism for the computation of these parameters $[63,64,71]$. To this end, we use the analytic formulae outlined in [63]. We first do a consistency check of the results that we discussed after solving the Boltzmann equations with those obtained by the analytic formulae. Then we briefly discuss the overall implementation procedure of the analytic solutions that will be followed in the context of the concerned model.

Solutions to eq. (3.1) and (3.2) can be written as [60]

$$
N_{B-L}^{\text {lepto }}=-\sum_{i}^{2} \varepsilon_{i} \kappa_{i},
$$

where $\kappa_{i}$ is the efficiency of the asymmetry production due to the $i^{\text {th }} \mathrm{RH}$ neutrino and is given by

$$
\kappa_{i}(z)=-\int_{z_{\mathrm{in}} \rightarrow 0}^{z_{\mathrm{fin}} \rightarrow \infty} \frac{d N_{N_{i}}}{d z^{\prime}} e^{-\sum_{i} \int_{z^{\prime}}^{z} W_{i}^{\mathrm{ID}}\left(z^{\prime \prime}\right) d z^{\prime \prime}} d z^{\prime} .
$$

For a strong washout regime, $\frac{d N_{N_{i}}}{d z^{\prime}} \simeq \frac{d N_{N_{i}}^{\text {eq }}}{d z^{\prime}}$, since the Yukawa couplings are strong enough to let any species of $N_{i}$ reach the equilibrium density, even if one starts from vanishing thermal abundance. One has to compare the $\kappa_{i}(z \rightarrow \infty)$, obtained by solving eq. (3.13) numerically, with the efficiency factor $\kappa_{i}^{\infty}$, obtained for a pure $N_{1}$ or $N_{2}$ dominated scenario, calculated at $z \rightarrow \infty$ and for thermal initial abundances of the RH neutrinos [63], ${ }^{6}$

$$
\begin{aligned}
\kappa_{1}^{\infty} & =\frac{2}{K_{1} z_{B}\left(K_{1}\right)}\left(1-e^{-\frac{K_{1} z_{B}\left(K_{1}\right)}{2}}\right), \\
\kappa_{2}^{\infty} & =\frac{2}{K_{2} z_{B}\left(K_{2}\right)}\left(1-e^{-\frac{K_{2} z_{B}\left(K_{2}\right)}{2}}\right) e^{-\int_{0}^{\infty} W_{1}^{\mathrm{ID}}(z) d z}, \\
& =\frac{2}{K_{2} z_{B}\left(K_{2}\right)}\left(1-e^{-\frac{K_{2} z_{B}\left(K_{2}\right)}{2}}\right) e^{-3 \pi K_{1} / 8},
\end{aligned}
$$

where

$$
z_{B}\left(K_{i}\right)=2+4 K_{i}^{0.13} e^{-\frac{2.5}{K_{i}}}
$$

To arrive at the exponential washout of $\kappa_{2}^{\infty}$ by $N_{1}$, we use

$$
\int_{0}^{\infty} z^{\alpha-1} \mathcal{K}_{n}(z) d z=2^{\alpha-2} \Gamma\left(\frac{\alpha-n}{2}\right) \Gamma\left(\frac{\alpha+n}{2}\right) .
$$

In figure 2, we show the comparison between $\kappa_{1}$ and $\kappa_{1}^{\infty}$ for two different values of $K_{1,2} \in(15,25)$. We find that for $K_{1,2}=25$, there is an excellent match between $\kappa_{1}$ and $\kappa_{1}^{\infty}$ for $\delta_{12} \geq 1$, which is consistent with the conclusions drawn in figure 1 (top-left). However, as expected, when one considers a lower value for $K_{1}$, say $K_{1}=15$, it is no longer safe to use $\delta_{12}=1$ for a hierarchical $N_{1}$ DS.

\footnotetext{
${ }^{6}$ In any case, for strong washout regime, final asymmetry does not depend upon initial conditions, e.g., see $[63,64]$.
} 


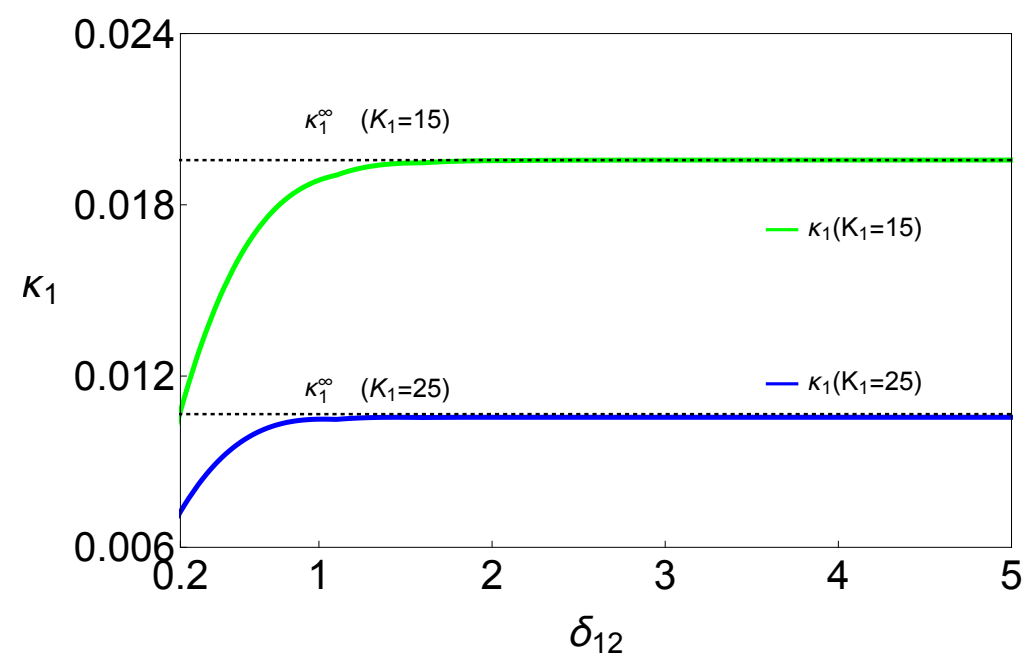

Figure 2. Efficiency factor with $\delta_{12}$ for two different values of $K_{1}$ with a fixed value of $K_{2}=25$.

It is also useful to have an expression for the efficiency factor for a strong washout scenario and any value of $\delta_{12}$. In this context, one can use [98]

$$
\kappa_{1}^{\mathrm{fit}}=\frac{2 K_{1}}{z_{B}\left(K_{1}+K_{2}^{\left(1-\delta_{12}\right)^{3}}\right)\left(K_{1}+K_{2}^{1-\delta_{12}}\right)},
$$

to scan the model, and estimate the minimum hierarchy of the $\mathrm{RH}$ neutrino masses for which $\kappa_{1}^{\text {fit }} \rightarrow \kappa_{1}^{\infty}$. To quantify the goodness of this estimate, one can define an error function given by

$$
\operatorname{Err}=\left|\frac{\kappa_{1}^{\mathrm{fit}}-\kappa_{1}^{\infty}}{\kappa_{1}^{\infty}}\right| \times 100 \% .
$$

In figure 3 (left panel), we show the error function for the two discussed cases, $\delta_{12}=1$ and $\delta_{12}=3$. It is obvious from this figure, that for the values of $\delta_{12}=1$ and $\delta_{12}=3$ chosen in figure 1 , the scope of error is always less that $\mathcal{O}(10 \%)$. In right panel of figure 3 , we show the comparison between $\kappa_{1}^{\text {fit }}$ and $\kappa_{1}^{\infty}$ for the given values of $\delta_{12}$ in the strong washout regime. Clearly, if $\delta_{12}=0.3$ (blue dashed line), one needs larger values for the decay parameter $K_{1}$ to circumvent the washout effect by $N_{2}$. Therefore, eq. (3.18) is also a reasonably good analytic approximation that can be used in the computation.

Thus, given the ranges of $K_{1}$ and $K_{2}$, one can do a random scanning over $\delta_{12}$ for each pair of $K_{1,2}$ to compare $\kappa_{1}(z)$ of eq. (3.13) or $\kappa_{1}^{\text {fit }}$ of eq. (3.18) to $\kappa_{1}^{\infty}$ upto desired accuracy, and extract the minimum values of $\delta_{12}$ needed to probe a perfectly valid hierarchical $N_{1}$ DS. We shall show in the next section that lowering the value of $\delta_{12}$ has two major consequences. Firstly, for low values of $\delta_{12}$, one enhances the CP asymmetry parameter, which in turn increases the magnitude of the asymmetry due to an enhancement in the loop functions (particularly in the self energy contribution [61]). Secondly, when flavour effects are accounted for, the contribution from $N_{2}[78,95]$ to the final asymmetry plays an important role in a successful leptogenesis. Thus, given a particular flavour regime, 

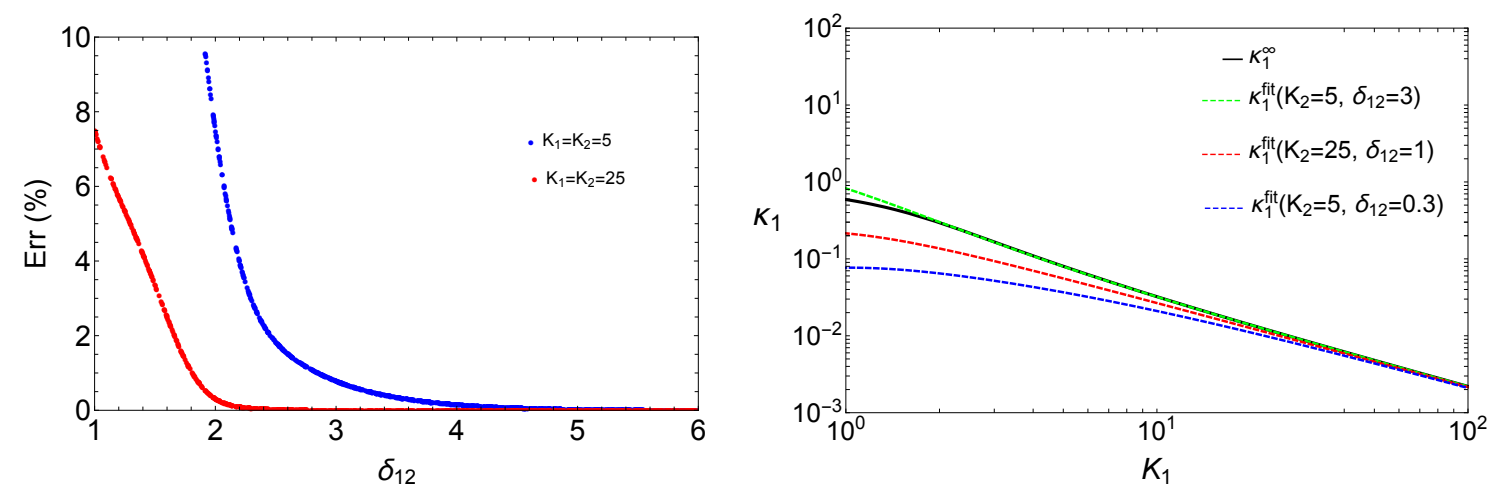

Figure 3. Left: possible error due to $\kappa_{1}^{\text {fit }}$ as the efficiency factor in a hierarchical scenario, as a function of $\delta_{12}$. Right: comparision of $\kappa_{1}^{\text {fit }}$ to $\kappa_{1}^{\infty}$ for different values of $\delta_{12}$ and $K_{2}$.

lowering the value of $\delta_{12}$ enables us to extract information regarding $N_{2}$-leptogenesis over a wide range of $\mathrm{RH}$ neutrino mass scale.

\section{Flavour effects and importance of $N_{2}$-leptogenesis}

The one flavour regime (1FR) is typically characterised by $M_{i}>10^{12} \mathrm{GeV}$ where all the charged lepton flavours are out of equilibrium, and thus the lepton doublet $\left|\ell_{i}\right\rangle$ produced by the decay of the RH neutrinos can be written as a coherent superposition of the corresponding flavour states $\left|\ell_{\alpha}\right\rangle$ as,

$$
\begin{array}{ll}
\left|\ell_{i}\right\rangle=\mathcal{A}_{i \alpha}\left|\ell_{\alpha}\right\rangle & (i=1,2,3 ; \alpha=e, \mu, \tau) \\
\left|\bar{\ell}_{i}\right\rangle=\overline{\mathcal{A}}_{i \alpha}\left|\bar{\ell}_{\alpha}\right\rangle & (i=1,2,3 ; \alpha=e, \mu, \tau),
\end{array}
$$

where the amplitudes are given by

$$
\mathcal{A}_{i \alpha}^{0}=\frac{m_{D_{i \alpha}}}{\sqrt{\left(m_{D} m_{D}^{\dagger}\right)_{i i}}} \quad \text { and } \quad \overline{\mathcal{A}}_{i \alpha}^{0}=\frac{m_{D_{i \alpha}}^{*}}{\sqrt{\left(m_{D} m_{D}^{\dagger}\right)_{i i}}} .
$$

Since there is hardly any interaction to break the coherence of the quantum states before it inversely decays to $N_{1}$, the asymmetry will be produced along the direction of $\left|\ell_{i}\right\rangle$ (or $\left.\left|\bar{\ell}_{i}\right\rangle\right)$ in the flavour space. However, this is not the case if $M_{i}<10^{12} \mathrm{GeV}$, since below this scale, flavour effects become important. We give a brief overview of the flavour effects at play during leptogenesis in this section.

The flavour effects are taken into account by defining the branching ratios into individual flavours as $P_{i \alpha}=\left|\mathcal{A}_{i \alpha}\right|^{2}$ and $\bar{P}_{i \alpha}=\left|\overline{\mathcal{A}}_{i \alpha}\right|^{2}$. As a result, the decays into individual flavours could be written as $\Gamma_{i \alpha} \equiv P_{i \alpha} \Gamma_{i}$ and $\bar{\Gamma}_{i \alpha} \equiv \bar{P}_{i \alpha} \bar{\Gamma}_{i}$ with $\sum_{\alpha}\left(P_{i \alpha}, \bar{P}_{i \alpha}\right)=1$. It is also convenient to introduce the flavoured decay parameter $K_{i \alpha}$ given by

$$
K_{i \alpha}=\frac{\Gamma_{i \alpha}+\bar{\Gamma}_{i \alpha}}{H\left(T=M_{i}\right)} \simeq \frac{P_{i \alpha}^{0}\left(\Gamma_{i}+\bar{\Gamma}_{i}\right)}{H\left(T=M_{i}\right)} \equiv P_{i \alpha}^{0} K_{i} \equiv \frac{\left|m_{D_{i \alpha}}\right|^{2}}{M_{i} m^{*}}
$$


where $m^{*} \simeq 10^{-3} \mathrm{eV}$ is the equilibrium neutrino mass. These flavoured probabilities can be re-written as

$$
\begin{aligned}
& P_{i \alpha}=P_{i \alpha}^{0}+\frac{\Delta P_{i \alpha}}{2}, \\
& \bar{P}_{i \alpha}=P_{i \alpha}^{0}-\frac{\Delta P_{i \alpha}}{2},
\end{aligned}
$$

where

$$
\begin{aligned}
P_{i \alpha}^{0} & =\frac{1}{2}\left(P_{i \alpha}+\bar{P}_{i \alpha}\right), \\
\Delta P_{i \alpha} & =P_{i \alpha}-\bar{P}_{i \alpha}
\end{aligned}
$$

are the tree level projectors. Here $\Delta P_{i \alpha}$, the difference between the tree level and the loop level projectors, arises from the fact that $\mathcal{A}_{i \alpha} \neq \overline{\mathcal{A}}_{i \alpha}$ [64], except at tree level. This allows us to define the flavoured $\mathrm{CP}$ asymmetry parameter $\varepsilon_{i \alpha}$ (see eq. (3.3)) as

$$
\varepsilon_{i \alpha}=P_{i \alpha}^{0} \varepsilon_{i}+\Delta P_{i \alpha} / 2 .
$$

Thus, due to the incorporation of flavour effects, an extra amount of CP violation, characterised by $\Delta P_{i \alpha}$, is generated. Note that in eq. (4.9) one can have CP violation in each flavour even if the total CP asymmetry is vanishing [99]. Typically, the effect of $\Delta P_{i \alpha}$ can be neglected in the washout terms, however, this is not the case for $\varepsilon_{i \alpha}$.

In the regime $10^{9} \mathrm{GeV}<M_{i}<10^{12} \mathrm{GeV}$, the $\tau$ flavored lepton comes into equilibrium, thereby breaking the coherent evolution of $\left|\ell_{i}\right\rangle$ before it inverse decays to $N_{i}$. As a result, $\left|\ell_{i}\right\rangle$ is projected onto a two flavour basis, characterised by the eigenstates along the directions of $\tau$, and perpendicular to it $\left(\tau_{i}^{\perp}\right)$, which is essentially a coherent superposition of the $\mu$ and the $e$ flavour. In the three flavour regime, i.e. all $M_{i}<10^{9} \mathrm{GeV}$, the $\mu$ lepton also comes into equilibrium, thus breaking the coherent evolution of the states along $\tau_{i}^{\perp}$. This allows for the individual resolution of all the flavours. Thus, calculating the asymmetry produced requires tracking the lepton asymmetry in the relevant flavours.

For example, in the $2 \mathrm{FR}$, the lepton asymmetry has to be tracked in $\tau$ and $\tau_{i}^{\perp}$. The Boltzmann equations can be written as

$$
\begin{aligned}
& \frac{d N_{N_{i}}}{d z}=-D_{i}\left(N_{N_{i}}-N_{N_{i}}^{\mathrm{eq}}\right), \text { with } i=1,2 . \\
& \frac{d N_{\Delta_{\alpha}}}{d z}=-\sum_{i=1}^{2} \varepsilon_{i \alpha} D_{i}\left(N_{N_{i}}-N_{N_{i}}^{\mathrm{eq}}\right)-\sum_{i=1}^{2} P_{i \alpha}^{0} W_{i}^{\mathrm{ID}} N_{\Delta_{\alpha}} .
\end{aligned}
$$

The asymmetry in the flavour $\alpha$ is given by

$$
N_{\Delta_{\alpha}}=-\sum_{i}^{2} \varepsilon_{i \alpha} \kappa_{i \alpha},
$$

with the efficiency factor

$$
\kappa_{i \alpha}(z)=-\int_{z_{\text {in }}}^{\infty} \frac{d N_{N_{i}}}{d z^{\prime}} e^{-\sum_{j} \int_{z^{\prime}}^{z} P_{j \alpha}^{0} W_{j}^{\mathrm{ID}}\left(z^{\prime \prime}\right) d z^{\prime \prime}} d z^{\prime} .
$$




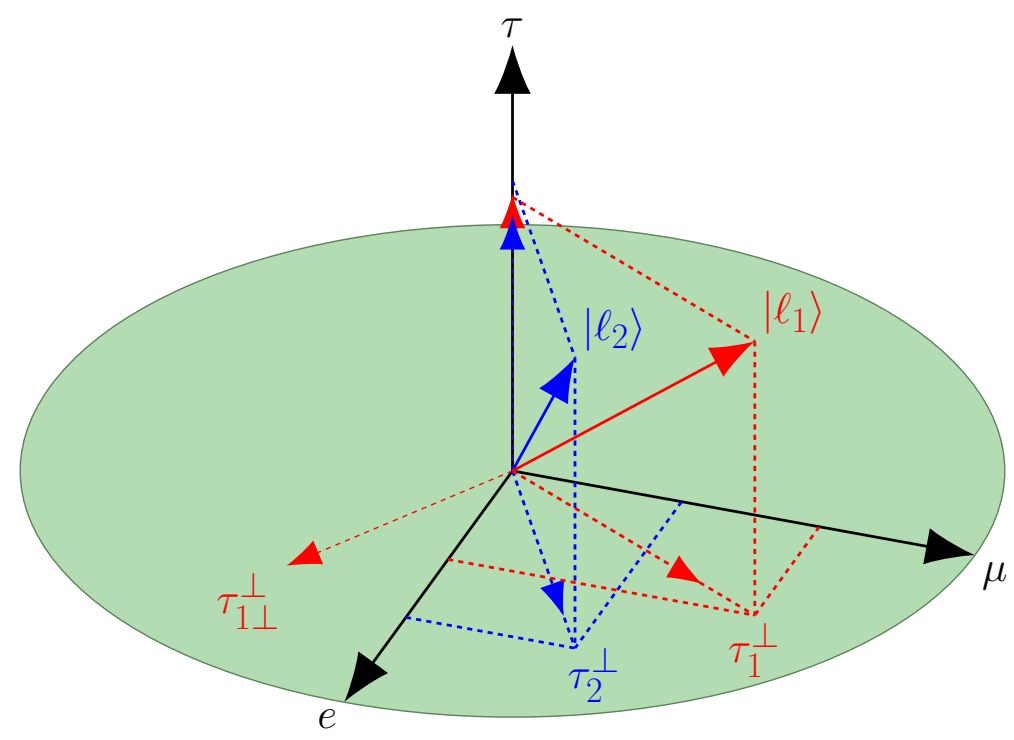

Figure 4. Ilustration of the two flavour regime for two RH neutrino model.

With this definition, the final baryon to photon ratio is

$$
\eta_{B}=0.96 \times 10^{-2} \sum_{\alpha} N_{\Delta_{\alpha}} .
$$

In the hierarchical limit of the RH neutrino masses, eq. (4.12) can be simplified as

$$
N_{\Delta_{\alpha}}=-\varepsilon_{1 \alpha} \kappa_{1 \alpha}^{\infty}-\varepsilon_{2 \alpha} \kappa_{2 \alpha}^{\infty} e^{-3 \pi K_{1 \alpha} / 8}
$$

where the first term is the asymmetry generated by $N_{1}$, and the second term is the asymmetry generated by $N_{2}$, subjected to $N_{1}$-washout. However, there are two important issues, which are usually overlooked in the leptogenesis studies of models with flavour symmetries.

(i) A pure $N_{1}$-leptogenesis scenario which is studied in most of the neutrino mass models, requires large values of the $N_{1}$ decay parameter $K_{1 \alpha}$ to washout the contribution from $N_{2}$. Thus given a neutrino mass model constrained by $3 \sigma$ oscillation data, one has to check the strength of $K_{1 \alpha}$ so that the second term of eq. (4.15) can be neglected.

(ii) Most importantly, if the masses of both the $\mathrm{RH}$ neutrinos are in the $2 \mathrm{FR}$, i.e., $10^{9} \mathrm{GeV}$ $<M_{i}<10^{12} \mathrm{GeV}$, after the $\tau$-interactions of both the states $\left|\ell_{1}\right\rangle$ and $\left|\ell_{2}\right\rangle$, the resultant states orthogonal to the $\tau$ flavour will not be in the same direction on the $e-\mu$ plane. This is demonstrated in figure 4 , where the new directions are denoted by $\tau_{1}^{\perp}$ and $\tau_{2}^{\perp}$ respectively. This is simply due the fact that, in general $\mathcal{A}_{1 \alpha} \neq \mathcal{A}_{2 \alpha}$, and hence, there is no reason for the states to maintain a common direction.

Henceforth, we denote the $\tau_{i}^{\perp}$ states as $\left|\ell_{1}^{\tau^{\perp}}\right\rangle$ and $\left|\ell_{2}^{\tau^{\perp}}\right\rangle$, which are given by

$$
\begin{aligned}
\left|\ell_{1}^{\perp}\right\rangle & =\frac{\mathcal{A}_{1 e}}{\sqrt{\left|\mathcal{A}_{1 e}\right|^{2}+\left|\mathcal{A}_{1 \mu}\right|^{2}}}\left|\ell_{e}\right\rangle+\frac{\mathcal{A}_{1 \mu}}{\sqrt{\left|\mathcal{A}_{1 e}\right|^{2}+\left|\mathcal{A}_{1 \mu}\right|^{2}}}\left|\ell_{\mu}\right\rangle, \\
\left|\ell_{2}^{\perp}\right\rangle & =\frac{\mathcal{A}_{2 e}}{\sqrt{\left|\mathcal{A}_{2 e}\right|^{2}+\left|\mathcal{A}_{2 \mu}\right|^{2}}}\left|\ell_{e}\right\rangle+\frac{\mathcal{A}_{2 \mu}}{\sqrt{\left|\mathcal{A}_{2 e}\right|^{2}+\left|\mathcal{A}_{2 \mu}\right|^{2}}}\left|\ell_{\mu}\right\rangle .
\end{aligned}
$$


In order to guess how much asymmetry generated by $N_{2}$ along $\tau_{2}^{\perp}$ can be washed out by the interactions between the Higgs and the component of $\left|\ell_{2}^{\tau^{\perp}}\right\rangle$ along $\left|\ell_{1}^{\tau^{\perp}}\right\rangle\left(N_{1}\right.$ inverse decay), one has to calculate the probability of $\left|\ell_{2}^{\tau^{\perp}}\right\rangle$ being in the $\left|\ell_{1}^{\tau^{\perp}}\right\rangle$ state. Note that for the $N_{1}$ inverse decay, only $\left(\left\langle\ell_{1}^{\tau^{\perp}} \mid \ell_{2}^{\tau^{\perp}}\right\rangle\right)\left|\ell_{1}^{\tau^{\perp}}\right\rangle$ will interact with the Higgs, whereas $\left(\left\langle\ell_{1 \perp}^{\tau^{\perp}} \mid \ell_{2}^{\tau^{\perp}}\right\rangle\right)\left|\ell_{1 \perp}^{\tau^{\perp}}\right\rangle$, which is perpendicular to $\left|\ell_{1}^{\tau^{\perp}}\right\rangle$, will be blind to it. Thus, the asymmetry in the direction of $\left|\ell_{1 \perp}^{\tau_{\perp}^{\perp}}\right\rangle$ will escape the $N_{1}$ washout and survive as a pure contribution from $N_{2}$.

The overlap probability $p_{12}$ can be calculated as

$$
p_{12} \equiv\left|\left\langle\ell_{1}^{\tau^{\perp}} \mid \ell_{2}^{\tau^{\perp}}\right\rangle\right|^{2}=\frac{K_{1} K_{2}}{K_{1 \tau^{\perp}} K_{2 \tau^{\perp}}} \frac{\left|\left(m_{D}^{*}\right)_{1 e}\left(m_{D}\right)_{2 e}+\left(m_{D}^{*}\right)_{1 \mu}\left(m_{D}\right)_{2 \mu}\right|^{2}}{h_{11} h_{22}},
$$

where $h_{i i}=\left(m_{D} m_{D}^{\dagger}\right)_{i i}$.

With this understanding, the r.h.s. of eq. (4.15) can be split into three parts

$$
\begin{aligned}
N_{\Delta_{\tau}} & =-\varepsilon_{1 \tau} \kappa_{1 \tau}^{\infty}-\varepsilon_{2 \tau} \kappa_{2 \tau}^{\infty} e^{-3 \pi K_{1 \tau} / 8}, \\
N_{\Delta_{\tau_{1}^{\perp}}} & =-\varepsilon_{1 \tau^{\perp}} \kappa_{1 \tau^{\perp}}^{\infty}-p_{12} \varepsilon_{2 \tau^{\perp}} \kappa_{2 \tau^{\perp}}^{\infty} e^{-3 \pi K_{1 \tau^{\perp}} / 8}, \\
N_{\Delta_{\tau_{\perp \perp}}} & =-\left(1-p_{12}\right) \varepsilon_{2 \tau^{\perp}} \kappa_{2 \tau^{\perp}}^{\infty},
\end{aligned}
$$

where the final $B-L$ asymmetry is given by

$$
N_{B-L}^{f}=N_{\Delta_{\tau}}+N_{\Delta_{\tau_{1}^{\perp}}}+N_{\Delta_{\tau_{1 \perp}^{\perp}}}
$$

Note that in a situation where a strong washout by the $N_{1}$ inverse decay prevails, the second term in eq. (4.19) and (4.20) can be dropped. Hence, the $p_{12} \rightarrow 1$ would imply a pure $N_{1}$-leptogenesis. In the literature, along with a strong $N_{1}$-washout, it is usually assumed that $p_{12}=1$, which is not true in general.

Another interesting situation arises when $M_{2}$ is in the two flavour regime and $M_{1}$ is in the three flavour regime. In this case, the produced asymmetry by $N_{2}$ in two flavour regime will be washed out by $N_{1}$ in the three flavour regime. Therefore, at the end of $N_{1}$-washout, we need to track the final asymmetry in individual flavours $(e, \mu, \tau)$. Thus, the asymmetry in each flavour can be written as

$$
\begin{aligned}
& N_{\Delta_{\tau}}=-\varepsilon_{1 \tau} \kappa_{1 \tau}^{\infty}-\varepsilon_{2 \tau} \kappa_{2 \tau}^{\infty} e^{-3 \pi K_{1 \tau} / 8}, \\
& N_{\Delta_{\mu}}=-\varepsilon_{1 \mu} \kappa_{1 \mu}^{\infty}-\frac{K_{2 \mu}}{K_{2 \tau^{\perp}}} \varepsilon_{2 \tau^{\perp}} \kappa_{2 \tau^{\perp}}^{\infty} e^{-3 \pi K_{1 \mu} / 8}, \\
& N_{\Delta_{e}}=-\varepsilon_{1 e} \kappa_{1 e}^{\infty}-\frac{K_{2 e}}{K_{2 \tau^{\perp}}} \varepsilon_{2 \tau^{\perp}} \kappa_{2 \tau^{\perp}}^{\infty} e^{-3 \pi K_{1 e} / 8},
\end{aligned}
$$

where the final $B-L$ asymmetry now is given by

$$
N_{B-L}=\sum_{\alpha} N_{\Delta_{\alpha}} \quad(\alpha=e, \mu, \tau) .
$$

Note that in eq. (4.23)-(4.25), the first term is the contribution to the final asymmetry from $N_{1}$ which produces the lepton asymmetry in $3 \mathrm{FR}$, where one can distinguish each of 


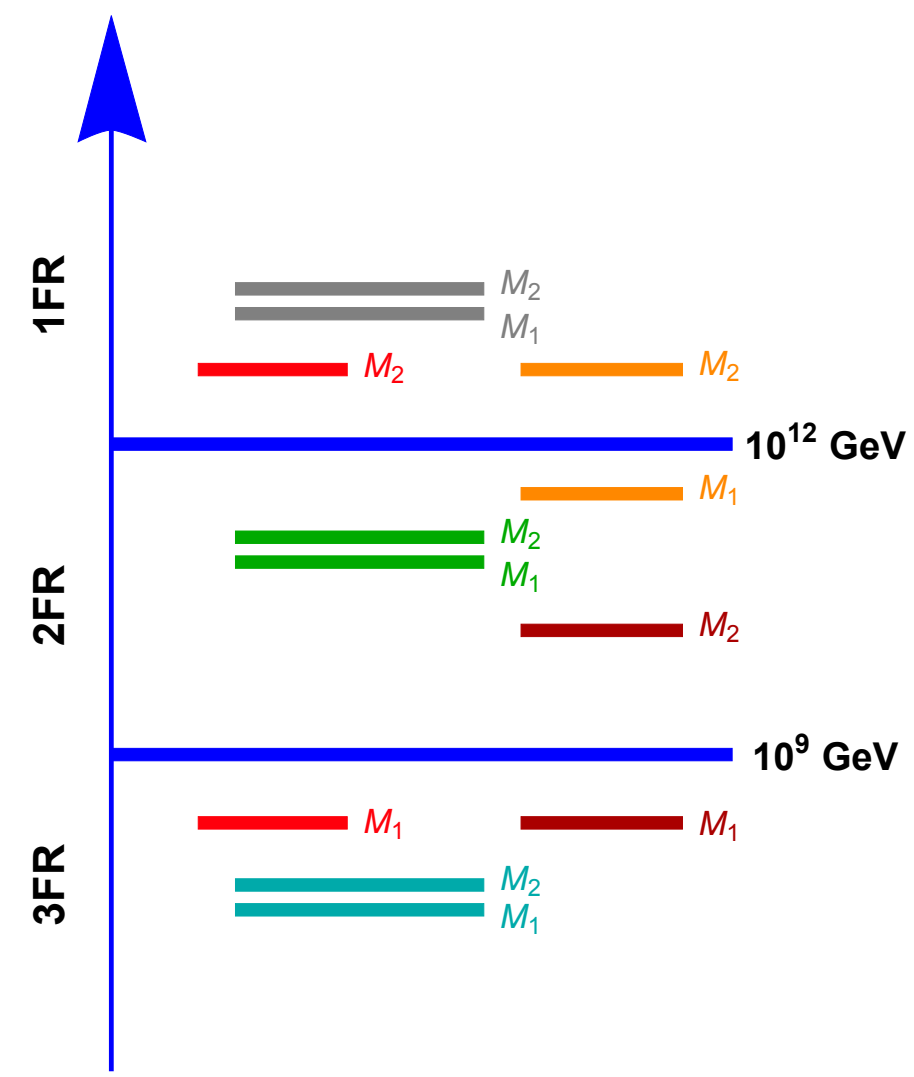

Allowed for $\mathrm{CP}^{\mu \tau}$

\section{Mass scale}

Figure 5. Various mass pattern in a leptogenesis scenario dominated by two right handed neutrinos. A particular RH neutrino mass which is either above $10^{12} \mathrm{GeV}$ or below $10^{9} \mathrm{GeV}$, can not generate baryon asymmetry in the $\mathrm{CP}^{\mu \tau}$ framework.

the three flavours. There could be other possibilities such as $M_{i}<10^{9} \mathrm{GeV}, M_{i}>10^{12}$, and $M_{2}>10^{12} \mathrm{GeV}$ but $M_{1}<10^{9} \mathrm{GeV}$ as shown in figure 5. Among these three possibilities, whilst the first one is not compatible to the standard thermal hierarchical leptogenesis scenario due to Davidson-Ibarra bound on $M_{i}$ [100], for the rest of the cases, successful leptogenesis cannot be realized unless we invoke some special conditions.

\section{Leptogenesis in the $\mathrm{CP}^{\mu \tau}$ symmetric model}

To carry out a numerical computation pertaining to a successful leptogenesis, we need to constrain the model parameters of eq. (2.12) with the present neutrino oscillation data [7]. For a normal neutrino mass ordering with solar and atmospheric mass squared differences, $\Delta m_{12}^{2}=7.39_{-0.20}^{+0.21} \times 10^{-5} \mathrm{eV}^{2}$ and $\Delta m_{31}^{2}=2.52_{-0.032}^{+0.033} \times 10^{-3} \mathrm{eV}^{2}$, the current global-fit values of the three mixing angle and the Dirac CP phases are tabulated in table 1. To this end, we follow the exact diagonalization procedure of a $3 \times 3$ light neutrino mass matrix, first demonstrated in [101]. This gives $-150^{\circ}<\theta<150^{\circ}$, while the ranges of the other parameters are shown in the figure 6 . 

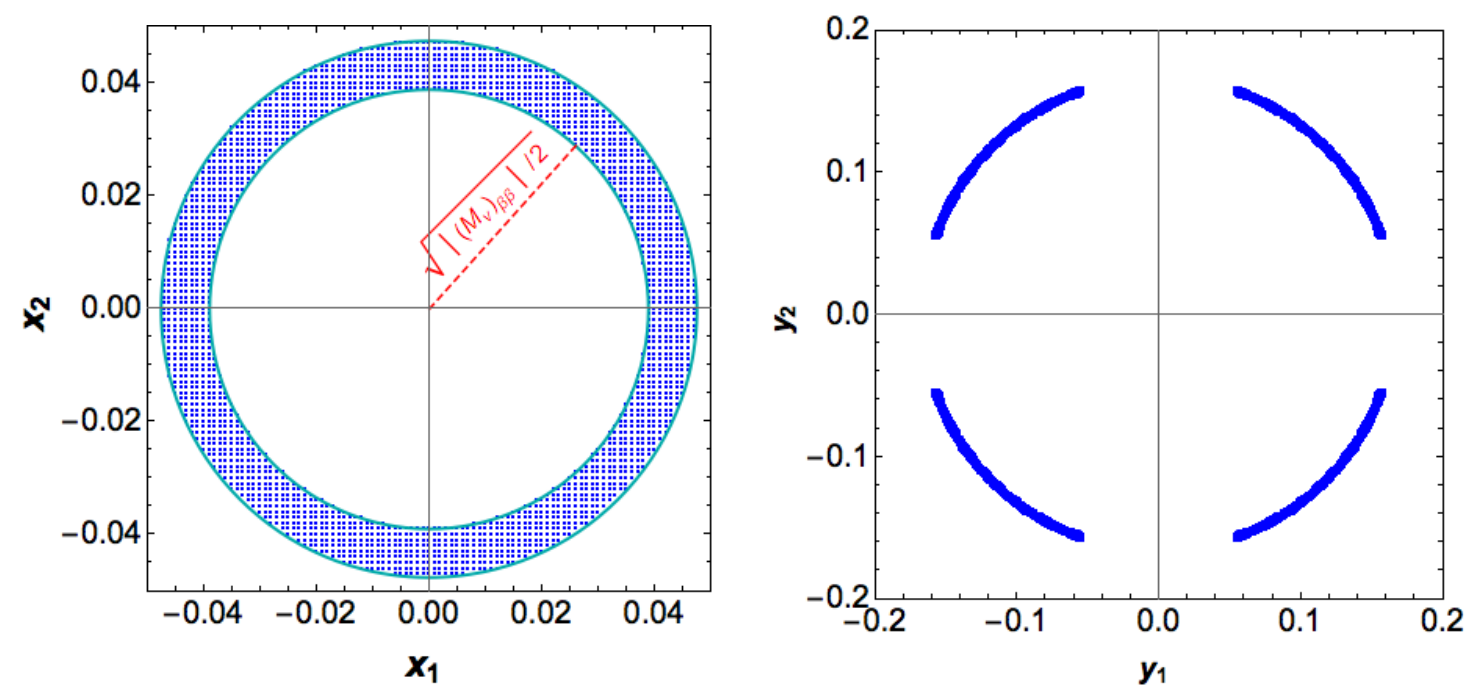

Figure 6. Parameter space of $\mathrm{CP}^{\mu \tau}$ symmetric mass matrix within a two $\mathrm{RH}$ neutrino scenario: $x_{1}$ vs $x_{2}$ (left) and $y_{1}$ vs $y_{2}$ (right), where these dimensional parameters (in $\sqrt{\mathrm{eV}}$ ) are defined in eq. (2.13).

\begin{tabular}{|c|cccc|}
\hline & $\theta_{12} /{ }^{\circ}$ & $\theta_{23} /{ }^{\circ}$ & $\theta_{13} /{ }^{\circ}$ & $\delta /{ }^{\circ}$ \\
\hline bf $\pm 1 \sigma$ & $33.82_{-0.76}^{+0.78}$ & $49.6_{-1.2}^{+1.0}$ & $8.61_{-0.13}^{+0.13}$ & $215_{-29}^{+40}$ \\
\hline $3 \sigma$ & $31.61 \rightarrow 36.27$ & $40.3 \rightarrow 52.4$ & $8.22 \rightarrow 8.99$ & $125 \rightarrow 392$ \\
\hline
\end{tabular}

Table 1. Best-fit, $1 \sigma$ and $3 \sigma$ ranges of three mixing angles and the Dirac CP phase $\delta$ for NMO $(\mathrm{NuFIT}[7])$.

The shape of the allowed parameter space in figure 6 could intuitively be inferred as follows. For a fixed value of $\left|\left(M_{\nu}\right)_{e e}\right|$ or $\left|\left(M_{\nu}\right)_{\mu \tau}\right|$ (say $c$ ), the solution is that of a circle, ${ }^{7}$ given by $x_{1}^{2}+x_{2}^{2}=c$ or $y_{1}^{2}+y_{2}^{2}=c$. Considering the left panel of figure 6 , since the radii of each of these circles are related to the neutrinoless double-beta decay parameter $\sqrt{\left|\left(M_{\nu}\right)_{\beta \beta}\right| / 2}$ (cf. eq. (2.12)), there exists an upper limit $\sim 5 \mathrm{meV}$ and a lower limit $\sim 3 \mathrm{meV}$ (represented by the cyan circles) on $\left|\left(M_{\nu}\right)_{\beta \beta}\right|$. However both the limits on $\left|\left(M_{\nu}\right)_{\beta \beta}\right|$ are beyond the sensitivity reach of the present experiments such as GERDA [102], KamLAND-Zen [103], EXO [104] etc., as well as the next generation experiments [105] like KamLAND2-Zen [106], nEXO [107], CUPID [108], CUORE [109], LEGEND-1k [110]. Thus, this model lacks testability from these experiments.

From eq. (2.12) and eq. (4.4), it is trivial to derive analytic correlations between the flavoured decay parameters as

$$
\begin{aligned}
& K_{2 e}=\frac{\left|\left(M_{\nu}\right)_{\beta \beta}\right|}{m^{*}}-K_{1 e} \\
& K_{2 \mu}=\frac{\left|\left(M_{\nu}\right)_{\mu \tau}\right|}{m^{*}}-K_{1 \mu}
\end{aligned}
$$

\footnotetext{
${ }^{7}$ Though it has been noticed that vanishing or close to vanishing values of $y_{1,2}$ are not compatible with present neutrino oscillation data. Discussion regarding the parameter sapce can be found in ref. [35].
} 

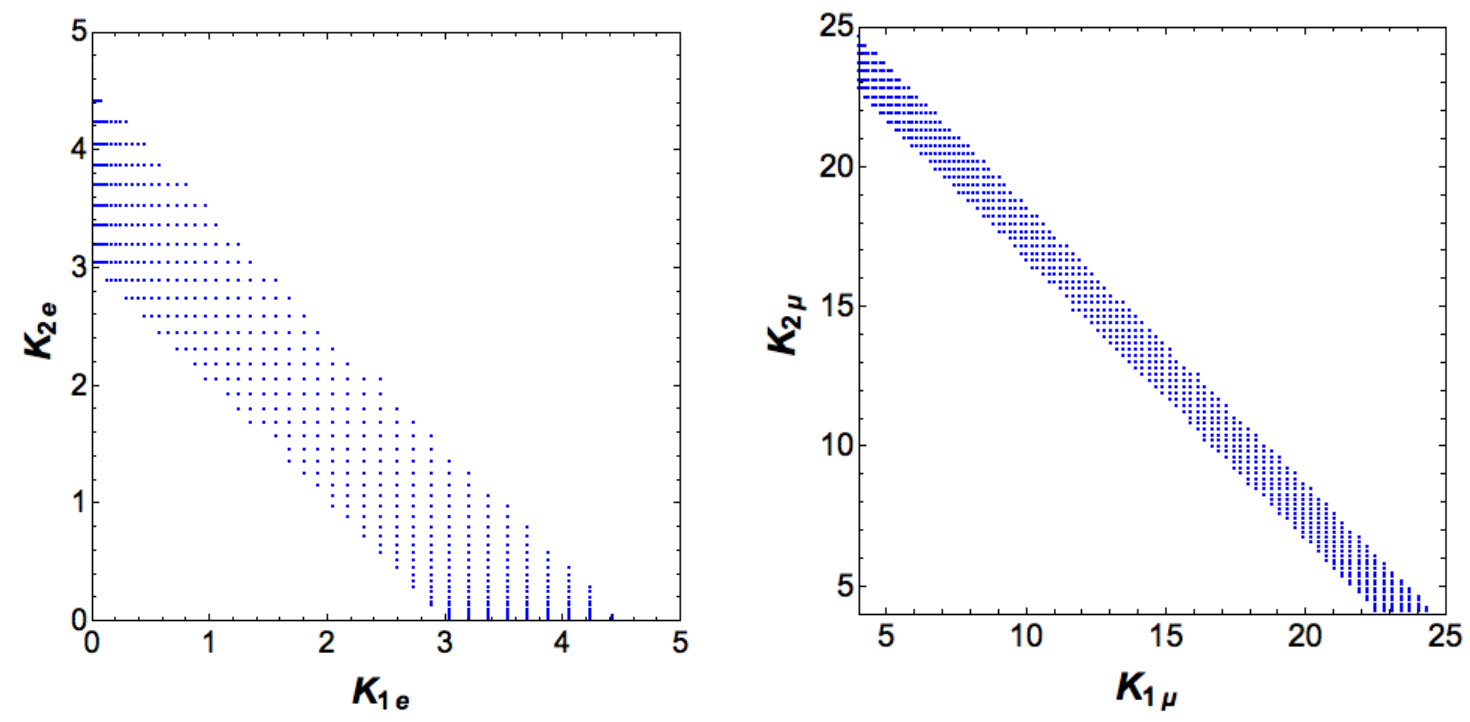

Figure 7. Flavoured decay parameters for both the RH neutrinos.

which are shown in figure 7 . There are two interesting observations to be made from these plots. Firstly, note that the decay parameters in the electron flavour can have approximately vanishing values, as is clear from the left panel. Secondly, the decay parameters in the muon flavour or tau flavour (in this case $K_{i \mu}=K_{i \tau}$ ) have a lower bound $(\sim 5)$ due to the discontinuity in parameter space of $y_{1}$ and $y_{2}$ (see right panel of figure 6 ). We see later that these ranges of the decay parameters have very interesting consequences on the process of leptogenesis in this model.

Let us first discuss two interesting mass patterns of the RH neutrinos: $M_{i}>10^{12} \mathrm{GeV}$ and $M_{i}<10^{9} \mathrm{GeV} .{ }^{8}$ First of all, for the one flavour regime $\left(M_{i}>10^{12} \mathrm{GeV}\right)$, the second term in eq. (3.4) vanishes when summed over ' $\alpha$ ', i.e, $\operatorname{Im}\left\{h_{j i}\left(m_{D}\right)_{i \alpha}\left(m_{D}^{*}\right)_{j \alpha}\right\}=\operatorname{Im}\left[\left|h_{j i}\right|^{2}\right]=$ 0 . The first term, however, is proportional to $\operatorname{Im}\left\{h_{i j}^{2}\right\}$. Using eq. (2.11), one can show that $h=m_{D} m_{D}^{\dagger}$ is a real matrix [35]. Thus, the flavour-summed CP asymmetry $\varepsilon_{i}=\sum_{\alpha} \varepsilon_{i \alpha}$ vanishes for any $i$. Therefore, successful leptogenesis is not possible in the unflavoured regime. Interestingly, $\varepsilon_{i e}$ is also vanishing, since the phases associated with the relevant parameters of $m_{D}$ will cancel when one uses eq. (3.4) to calculate the CP asymmetry in the electron flavour. Thus in this model, $\varepsilon_{i \mu} \equiv \Delta P_{i \mu} / 2=-\varepsilon_{i \tau}$. On the other hand, if all the RH neutrino masses are in the three flavour regime $M_{i}<10^{9} \mathrm{GeV}$, one might wonder whether there would be possibilities for a resonant leptogenesis [61, 62]. However, in [111], it has been analytically argued that due to the typical structure of the symmetry (the efficiency factors in $\mu$ and $\tau$ flavour are same), such a possibility still leads to a vanishing asymmetry even after taking into account the flavour coupling effects $[72,112,113]$.

Another interesting possibility is to consider $M_{2}>10^{12} \mathrm{GeV}$, and $M_{1}<10^{12} \mathrm{GeV}$. In that case, since the asymmetry is produced by $N_{2}$ in the unflavoured regime and

\footnotetext{
${ }^{8}$ Both these mass patterns have been discussed in literature, e.g., for the first one see [22, 69] and for the seconed one, see [111]. We recall the discussion here for comprehensiveness.
} 
$\varepsilon_{i}=\sum_{\alpha} \varepsilon_{i \alpha}=0$, the final baryon asymmetry only has contributions from $N_{1} \cdot{ }^{9}$ As a result, all the results derived in refs. [22,35] will be valid upto minor changes due to the newly released global-fit data [7].

In this paper, we shall focus on the following mass patterns: (i) $10^{9} \mathrm{GeV}<M_{1,2}<$ $10^{12} \mathrm{GeV}$, and (ii) $10^{9} \mathrm{GeV}<M_{2}<10^{12}$ and $M_{1}<10^{9} \mathrm{GeV}$. Before discussing these cases explicitly, we list the flavoured CP asymmetry parameters in this model. Using eq. (2.11) and eq. (3.4) the $\varepsilon_{i \alpha}$ can be obtained as

$$
\varepsilon_{i e}=0, \varepsilon_{i \mu}=-\xi_{i} \frac{g^{\prime}\left(x_{i j}\right)}{4 \pi v^{2}}\left[\frac{\left(a_{i} a_{j}+b_{i} b_{j} \cos \theta\right) b_{i} b_{j} \sin \theta}{a_{i}^{2}+b_{i}^{2}}\right]=-\varepsilon_{i \tau}, \quad i \neq j(=1,2),
$$

where $g^{\prime}\left(x_{i j}\right)$ is given by

$$
g^{\prime}\left(x_{i j}\right) \simeq\left[f\left(x_{i j}\right)+\sqrt{x_{i j}} /\left(1-x_{i j}\right)\right]+\left(1-x_{i j}\right)^{-1} \equiv g_{1}\left(x_{i j}\right)+g_{2}\left(x_{i j}\right),
$$

and $\xi_{i}= \pm 1$ for $i=1$ and 2 respectively. Using eq. (2.13) we can now simplify eq. (5.3) for $i=1$ as

$$
\varepsilon_{1}^{\mu}=-\frac{g^{\prime}\left(x_{12}\right) M_{2}}{4 \pi v^{2}}\left[\frac{\left(x_{1} x_{2}+y_{1} y_{2} \cos \theta\right) y_{1} y_{2} \sin \theta}{x_{1}^{2}+y_{1}^{2}}\right]=-\varepsilon_{1}^{\tau},
$$

which in the strong hierarchical limit can further be simplified as

$$
\varepsilon_{1}^{\mu} \simeq \frac{3 M_{1}}{8 \pi v^{2}}\left[\frac{\left(x_{1} x_{2}+y_{1} y_{2} \cos \theta\right) y_{1} y_{2} \sin \theta}{x_{1}^{2}+y_{1}^{2}}\right]=-\varepsilon_{1}^{\tau} .
$$

Similarly for $i=2$ the CP asymmetry parameter can be calculated as

$$
\varepsilon_{2}^{\mu}=\frac{g^{\prime}\left(x_{21}\right) M_{1}}{4 \pi v^{2}}\left[\frac{\left(x_{1} x_{2}+y_{1} y_{2} \cos \theta\right) y_{1} y_{2} \sin \theta}{x_{2}^{2}+y_{2}^{2}}\right]=-\varepsilon_{2}^{\tau} .
$$

Armed with these equations, we can proceed toward a systematic discussion of leptogenesis for the relevant cases.

\subsection{Two flavour regime: $10^{9} \mathrm{GeV}<M_{1,2}<10^{12} \mathrm{GeV}$}

The $N_{1}$-decay parameters in the muon and tau flavour are strong enough $[63,64]$ to washout any pre-existing asymmetry (see right panel of figure 7 ). Thus, all the terms which contain the exponential washout factors in eq. (4.19) and eq. (4.20) can be neglected. Therefore the total $N_{B-L}$ asymmetry can be written as

$$
\begin{aligned}
N_{B-L} & =-\left(\varepsilon_{1 \tau} \kappa_{1 \tau}^{\infty}+\varepsilon_{1 \tau^{\perp}} \kappa_{1 \tau^{\perp}}^{\infty}\right)-\left(1-p_{12}^{\perp}\right) \varepsilon_{2 \tau^{\perp}} \kappa_{2 \tau^{\perp}}^{\infty} \\
& =-\varepsilon_{1 \tau}\left(\kappa_{1 \tau}^{\infty}-\kappa_{1 \tau^{\perp}}^{\infty}\right)-\left(1-p_{12}^{\perp}\right) \varepsilon_{2 \mu} \kappa_{2 \mu}^{\infty},
\end{aligned}
$$

where we use the fact, that $\varepsilon_{i e}=0, \varepsilon_{i \mu}=-\varepsilon_{i \tau}$, and the electron decay parameters are much weaker than the muon decay parameters. Clearly, the first term in eq. (5.8), which is a contribution from $N_{1}$, is non-vanishing when $\kappa_{1 \tau}^{\infty} \neq \kappa_{1 \tau^{\perp}}^{\infty}$, i.e, when there is an asymmetric washout in the $\tau$ and $\tau^{\perp}$ flavour. The second term, driven by the muon flavour, is a pure

\footnotetext{
${ }^{9} N_{2}$ might contribute to the final asymmetry via phantom terms [78]. However, phantom leptogenesis in this context is beyond the scope of this study.
} 


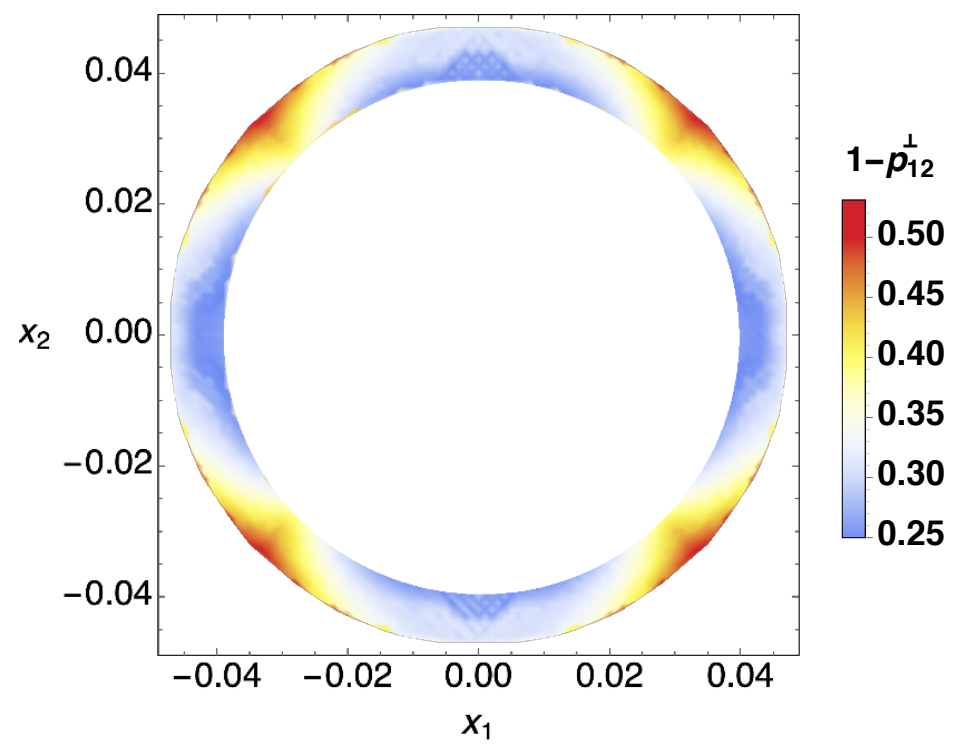

Figure 8. Plot showing the quantity $\left(1-p_{12}^{\perp}\right)$ with the model parameter $x_{1}$ and $x_{2}$. Since lepton asymmetry generated by $N_{2}$ is proportional to $\left(1-p_{12}^{\perp}\right)$, and clearly this never vanishes in this model, a pure $N_{1}$ dominated scenario is not possible. Note that $x_{1,2}$ are dimensional quantities, and are plotted in units of $\sqrt{\mathrm{eV}}$.

contribution from $N_{2}$, and is non-zero when $p_{12}^{\perp} \neq 1$. Using eq. (4.18), one can arrive at an expression for the probability $p_{12}^{\perp}$ as,

$$
p_{12}^{\perp}=\frac{4 x_{1}^{2} x_{2}^{2}+y_{1}^{2} y_{2}^{2}+4 x_{1} x_{2} y_{1} y_{2} \cos \theta}{\left(2 x_{1}^{2}+y_{1}^{2}\right)\left(2 x_{2}^{2}+y_{2}^{2}\right)} .
$$

In figure 8 we show the variation of $\left(1-p_{12}^{\perp}\right)$ with the model parameter $x_{1}$ and $x_{2}$. An interesting fact is that $\left(1-p_{12}^{\perp}\right)$ never vanishes in this model. This means $N_{2}$ always contributes to the final asymmetry. In addition, one has a strong concentration of points towards the higher values $(\sim 0.5)$ of $\left(1-p_{12}^{\perp}\right)$ which indicates there could be sizeable number of data points for which $N_{2}$ domination could be realized. In fact we show as we proceed, $N_{2}$ domination in this model is possible for a significant amount of parameter space $(\sim 26 \%)$.

We first concentrate on the choice of $\mathrm{RH}$ neutrino mass hierarchy in this model. To find the minimum value of $M_{2} / M_{1}$, we generalise the procedure described in section 3 and find that one may choose the RH neutrino mass hierarchy as mild as $M_{2} / M_{1} \sim 4.7$ for a perfectly valid $N_{1}$ DS. ${ }^{10}$ In the upper panel of figure 9 , we show the evolution of the $B-L$ asymmetry produced by both the $\mathrm{RH}$ neutrinos, in the two extreme cases of $K_{1 \tau}$ and $K_{2 \tau}$ (see right panel of figure 7 ). Note that, though for the first set of the decay parameters $\left(K_{2 \tau}=5\right.$ and $\left.K_{1 \tau}=25\right)$, hierarchical $N_{1}$ DS can be reproduced with $\delta_{12} \sim 1$, the second set $\left(K_{2 \tau}=25\right.$ and $\left.K_{1 \tau}=5\right)$ requires a larger value of $\delta_{12} \sim 3.7$. For the first case, the $N_{2}$-washout is not strong enough to affect the asymmetry production by $N_{1}$ up to very low values of $\delta_{12}(\sim 1)$. Thus for $\delta_{12} \geq 1$, the final dynamics is governed only by the

\footnotetext{
${ }^{10}$ We have checked this using eq. (3.13) as well as eq. (3.18).
} 

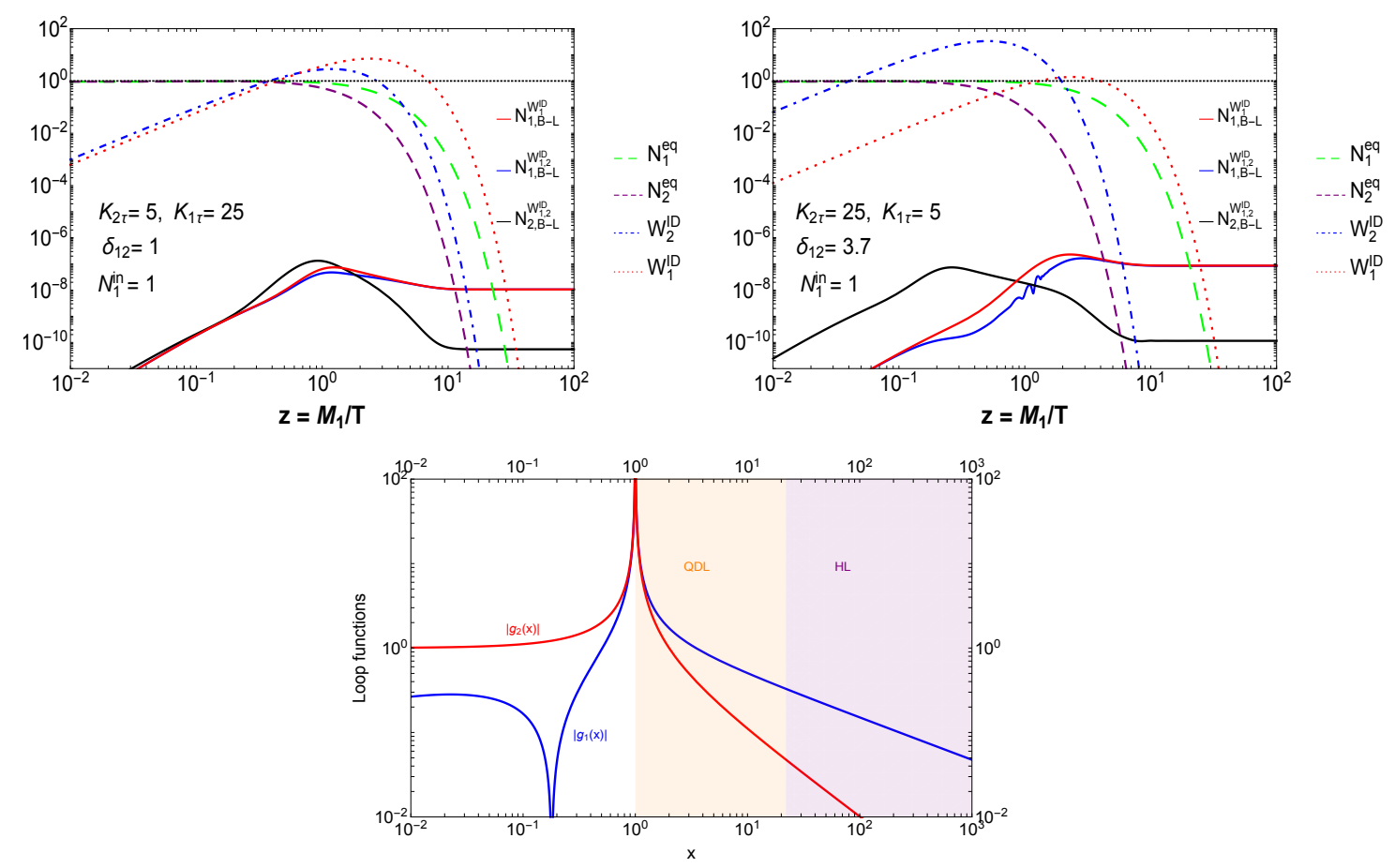

Figure 9. Upper panel: (Colour codes for the $N_{B-L}$ asymmetries are same as figure 1): representative plots showing the validity of $N_{1}$ dominated scenario when the decay parameter of $N_{2}$ is weaker than the decay parameters of $N_{1}$ (left), and vice-versa (right). Bottom panel: variation of the involved loop functions in the $\mathrm{CP}$ asymmetry parameters. The light violet region is the region where hierarchical scenario is valid in the model under consideration.

$N_{1}$-interactions. On the other hand, for the second case, the $N_{2}$-washout is much stronger and it starts to reduce the magnitude of the asymmetry produced by $N_{1}$, unless one goes beyond $\delta_{12} \geq 3.7$. Henceforth, we designate $\delta_{12}=3.7$ as the critical point which separates the hierarchical (HL) and quasi-degenerate limit (QDL) of leptogenesis in $\mathrm{CP}^{\mu \tau}$ model. We use this mild hierarchy criteria, i.e., $M_{2} / M_{1}=4.7$ in the computation of leptogenesis for rest of the paper.

Once we go from strong to a mild hierarchy, we immediately see an enhancement in the loop functions (cf. the bottom panel of figure 9). In hierarchical limit, it is sufficient to consider the enhancement in the function $g_{1}\left(x_{12}=M_{2}^{2} / M_{1}^{2}\right)$ which dominates in $\varepsilon_{1 \alpha}$. Due to this enhancement, the previously quoted lower bound on $M_{1}\left(\sim 6 \times 10^{10} \mathrm{GeV}\right)[22,35]$ gets lowered to $M_{1}^{\text {min }} \sim 7.5 \times 10^{9} \mathrm{GeV}$. Note that this can be further relaxed with the inclusion of flavour couplings, which tend to increase the efficiency of the asymmetry production. In addition, due to this choice of mild hierarchy $M_{2}$ would likely to be in the $2 \mathrm{FR}$ (the green rectangles in figure 5). However we stress that if one chooses a strong hierarchy, say $M_{2} / M_{1}=10^{3}([22,35,69]), M_{2}$ is necessarily in the $1 \mathrm{FR}$, if we take $M_{1}$ to be in the 2FR. Thus contribution from $M_{2}$ can be neglected since the total CP asymmetry vanishes in the unflavoured (1FR) regime. Therefore, the results obtained in the above references (for a pure $N_{1}$ domination) hold true. 


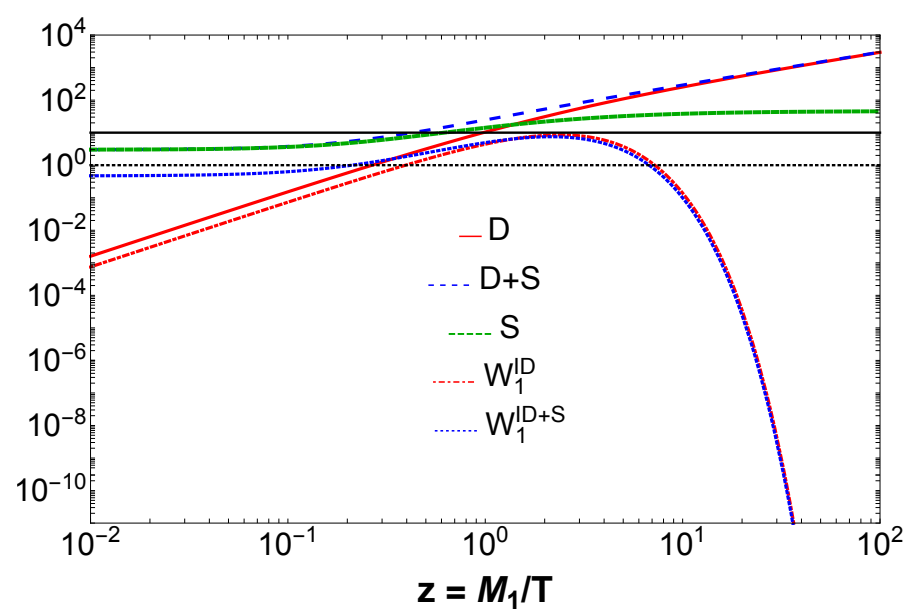

Figure 10. Comparison among rates of various processes involved in the leptogenesis. The horizontal black line is the rate of the $\tau$ charged lepton flavour interaction $\Gamma_{\tau} / H z$ at the $N_{1}$ leptogenesis temperature $T \sim M_{1} \sim 10^{11} \mathrm{GeV}$. Domination of $\Gamma_{\tau} / H z$ (over all the rates) has been considered to ensure a strongly decoherent picture for simplicity.

It is also worth mentioning that in this work, we consider a fully flavoured scenario where the charged lepton flavour interaction rate is dominant throughout the thermal history of the asymmetry production. Mathematically, this implies that the washout term $W\left(z^{\max }, K_{1}\right)<\Gamma_{\tau} / 2 H z$, where $\Gamma_{\tau}$ is the $\tau$ interaction rate. This condition translates into

$$
F_{\tau} \equiv \Gamma_{\tau} / 2 H z_{i}=\frac{5 \times 10^{11} \mathrm{GeV}}{M_{i}}>W\left(z_{i}^{\max }\right),
$$

where the washout term $W(z)$ contains inverse decays as well as dominant scattering rates (cf. figure 10). Notice that for a weak washout scenario, eq. (5.10) is trivially satisfied. In that case, the washout terms never reach equilibrium and thus, for any value of $M_{i}<5 \times 10^{11} \mathrm{GeV}$, the interaction rate $\Gamma_{\tau}$ is fast enough to break the coherence of the states produced by $N_{i}$. But for a strong washout, this is not the case since the washout term $W(z) \gg 1$. Thus, the masses for the RH neutrinos should be chosen carefully so that throughout the thermal history, $\Gamma_{\tau}$ dominates over the relevant washout rates. Otherwise, one needs to take into account the off-diagonal terms of the density matrix ${ }^{11}$ that account for the coherence among the basis states $[78,114,115]$. In this context, it is also worthwhile to recall refs. [116-119] that discuss leptogenesis using full quantum kinetic equations.

In the washout term, in addition to the inverse decay we include dominant $\Delta L=1$ scattering processes involving top quark. These processes include a combined contribution of the Higgs mediated $s$-channel $\left(N_{i} \ell \leftrightarrow q t\right)$ and $t$-channel processes $\left(N_{i} q \leftrightarrow \ell t\right)$. The relevant scattering rates for both the channels can be written as

$$
S_{\phi i}^{a}=\frac{\Gamma_{\phi i}^{a}}{H z}, \quad a=s, t .
$$

\footnotetext{
${ }^{11}$ Note that thus the CP asymmetry parameter defined in eq. (4.9) appears in the ' $\alpha \alpha$ ' (diagonal) term of the density matrix evolution equation [78, 115].
} 
The quantity $\Gamma_{\phi i}^{a}$ is related to the reaction density $\gamma_{\phi i}^{a}$ as $\Gamma_{\phi i}^{a}=\frac{\gamma_{\phi i}^{a}}{n_{N_{i}}^{\text {eq }}}$, where for the reaction density of a generic $2 \leftrightarrow 2$ process, one has the expression [64]

$$
\gamma(2 \leftrightarrow 2)=\frac{g_{x} g_{y} T}{32 \pi^{4}} \int d s s^{3 / 2} K_{1}(\sqrt{s} / T) \lambda\left(1, \frac{m_{x}^{2}}{s}, \frac{m_{y}^{2}}{s}\right) \sigma(s)^{a},
$$

where $g_{x}$ and $g_{y}$ are initial state degrees of freedom, $s$ is the center of mass energy and the quantity $\lambda$ is given by

$$
\lambda\left(1, \frac{m_{x}^{2}}{s}, \frac{m_{y}^{2}}{s}\right)=\left(1-\frac{m_{x}^{2}}{s}-\frac{m_{y}^{2}}{s}\right)^{2}-4 \frac{m_{x}^{2} m_{y}^{2}}{s^{2}} .
$$

The washout for the $\Delta L=1$ term could be written as

$$
W_{i}^{\Delta L=1}=W_{i}^{s}+2 W_{i}^{t},
$$

which are related to the scattering rate as

$$
W_{i}^{s}=\frac{N_{N_{i}}}{N_{\ell}^{\mathrm{eq}}} S_{\phi i}^{s}, W_{i}^{t}=\frac{N_{N_{i}}^{\mathrm{eq}}}{N_{\ell}^{\mathrm{eq}}} S_{\phi i}^{t} .
$$

We compare the total washout term $W=W_{i}^{\mathrm{ID}}+W_{i}^{\Delta L=1}$ with the charged lepton interaction rate $F_{\tau}$. Given the ranges of the decay parameters, we find that $M_{1} \sim 4 \times 10^{10} \mathrm{GeV}$ could be a safe value to circumvent the dominance of the washout processes over the charged lepton interaction. ${ }^{12}$ Notice that, for the mass window $M^{\max } \sim 4 \times 10^{10} \mathrm{GeV} \gtrsim M_{1} \gtrsim M_{1}^{\min } \sim$ $7.5 \times 10^{9}$, the formulae we use in this paper are technically valid. While the inclusion of flavour couplings could lower the value of $M_{1}^{\min }$ (as already pointed out before), one may still go beyond $M^{\max }$ and opt for a diagonal density-matrix formalism. However, in that case one has to neglect the higher values of the decay parameters (i.e., there would be upper bound on the decay parameters) which are responsible for the dominance of washout terms over the charged lepton interactions. In order to understand the contribution from $N_{1}$ and $N_{2}$ to $N_{B-L}$, we can write eq. (5.8) as

$$
N_{B-L}=-\varepsilon_{1 \tau}\left(\kappa_{1 \tau}^{\infty}-\kappa_{1 \tau^{\perp}}^{\infty}\right)-\left(1-p_{12}^{\perp}\right) \varepsilon_{2 \mu} \kappa_{2 \mu}^{\infty}=N_{B-L}^{N_{1}}+N_{B-L}^{N_{2}}
$$

where $N_{B-L}^{N_{1}}$ is the contribution from $N_{1}$ and $N_{B-L}^{N_{2}}$ is the contribution from $N_{2}$. The ratios

$$
R_{N_{1}}=\left|\frac{N_{B-L}^{N_{1}}}{N_{B-L}^{N_{2}}}\right|, \quad R_{N_{2}}=\left|\frac{N_{B-L}^{N_{2}}}{N_{B-L}^{N_{1}}}\right|
$$

can be used to realize a particular $N_{i}$ domination quantitatively. We use the criteria $R_{N_{i}}>10$ to signify a particular $N_{i}$ domination. Notice from figure 11 that indeed both the $R$ parameters can have values $\gg 10$; also, in general, $R_{N_{1}}>R_{N_{2}}$. Thus lepton asymmetry produced by both the neutrinos can dominate for certain region of the parameter space.

\footnotetext{
${ }^{12}$ In the numerical computaion we use $M_{\phi} / M_{1}=10^{-5}[120,121]$, where $M_{\phi}$ is the Higgs thermal mass needed to cut off the infrared divergences of $t$ channel process. For the scattering cross sections please see ref. [122].
} 

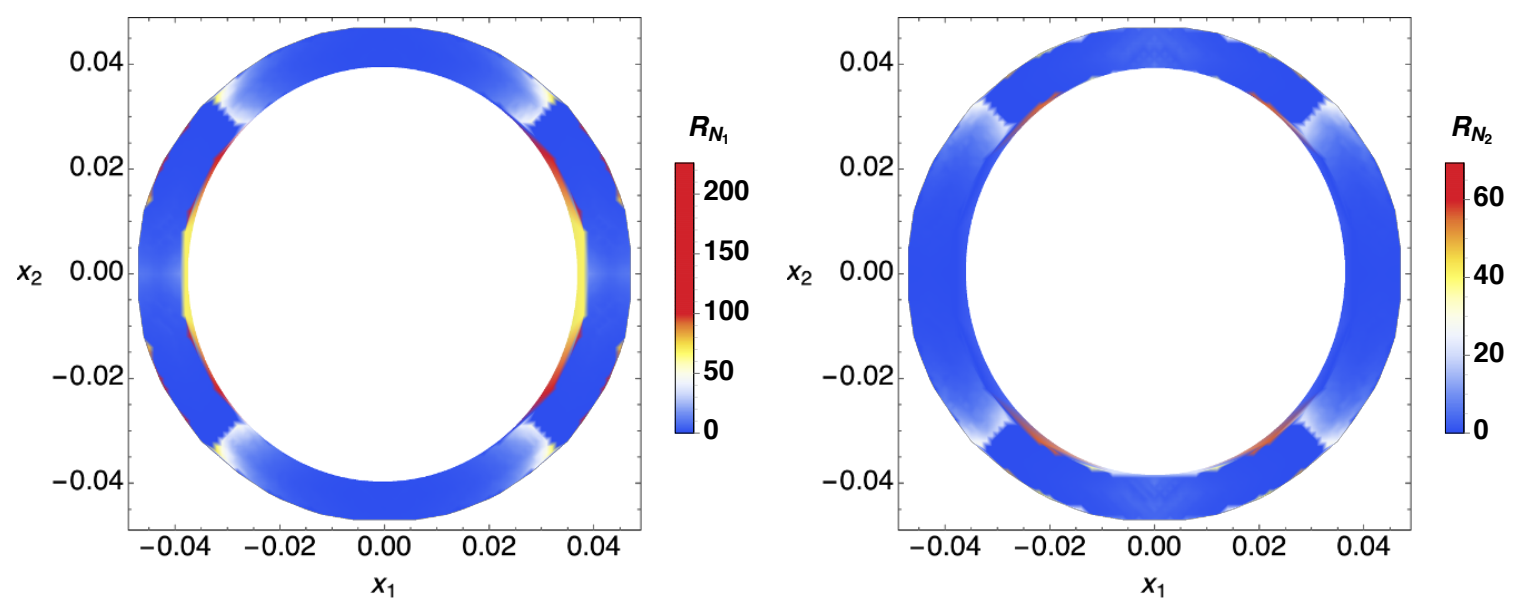

Figure 11. Top panel: $R_{N_{1}} \equiv \eta_{B}^{N_{1}} / \eta_{B}^{N_{2}}$ with the model parameters $x_{1}$ and $x_{2}$. Bottom panel: $R_{N_{2}} \equiv \eta_{B}^{N_{2}} / \eta_{B}^{N_{1}}$ with the model parameters $x_{1}$ and $x_{2}$. All the plots are generated for $M_{1}=4 \times 10^{10} \mathrm{GeV}$. Note that $x_{1,2}$ are dimensional quantities, and are plotted in units of $\sqrt{\mathrm{eV}}$.

Quantitatively, $37 \%$ of the parameter space favours a $N_{1}$ dominated scenario $\left(R_{N_{1}}>10\right)$ and $26 \%$ of the parameter space favours a $N_{2}$ dominated scenario $\left(R_{N_{2}}>10\right)$. These percentages have been calculated by taking the ratios of the number of data points corresponding to $R_{N_{i}}>10$ and the total number of data points compatible with $3 \sigma$ neutrino oscillation data. We stress that the above quantification is valid for any arbitrary values of $M_{1}$ in the mass window $4 \times 10^{10} \mathrm{GeV} \gtrsim M_{1} \gtrsim M_{1}^{\text {min }}$.

However, the real challenge is now to check whether the parameters corresponding to $R_{N_{1}}>10$ or $R_{N_{2}}>10$ are able to reproduce the observed range of the baryon to photon ratio. We checked that though the $N_{1}$ domination can be realized within the allowed mass window, $N_{2}$ domination can be realized marginally even if we take the maximal allowed value of $M_{1}^{\max } \sim 4 \times 10^{10} \mathrm{GeV}$. In figure 12 , we plot the baryon to photon ratio normalised to $6.3 \times 10^{-10}$ with the $R$ parameters for $M_{1}=7 \times 10^{10} \mathrm{GeV}$. Note that, though this value of $M_{1}$ is beyond $M_{1}^{\max }$, we do not lose any information on the $\mathrm{RH}$ neutrino masses by discarding the higher values of the decay parameters. Since higher values of $\eta_{B}$ correspond to lower values of the decay parameter, exclusion of higher values of the decay parameter implies truncating the lower portion of the parameter space (right hand side of figure 12) in the $R_{N_{2}}-\overline{\left|\eta_{B}\right|}$ plane which is anyway much below $\overline{\left|\eta_{B}\right|}=1$.

\section{$5.210^{9} \mathrm{GeV}<M_{2}<10^{12} \mathrm{GeV}$ and $M_{1}<10^{9} \mathrm{GeV}$}

In this section we give a qualitative picture of what happens in the case $10^{9} \mathrm{GeV}<M_{2}<$ $10^{12} \mathrm{GeV}$ and $M_{1}<10^{9} \mathrm{GeV}$. Two important points should be stressed a priori. Firstly, since the mass of $N_{1}$ is much less than $10^{9} \mathrm{GeV}$, the $\mathrm{CP}$ asymmetry parameter $\varepsilon_{1 \alpha}$ is highly suppressed and does not suffice to reproduce the correct baryon asymmetry [100]. One might wonder whether $N_{2}$ could produce a viable CP asymmetry or not. However, if we are in a two RH neutrino scenario (i.e., the third heavy neutrino does not couple to Higgs and leptons), the CP asymmetry parameter $\varepsilon_{2 \alpha}$ (cf eq. (5.7)) is also proportional to 

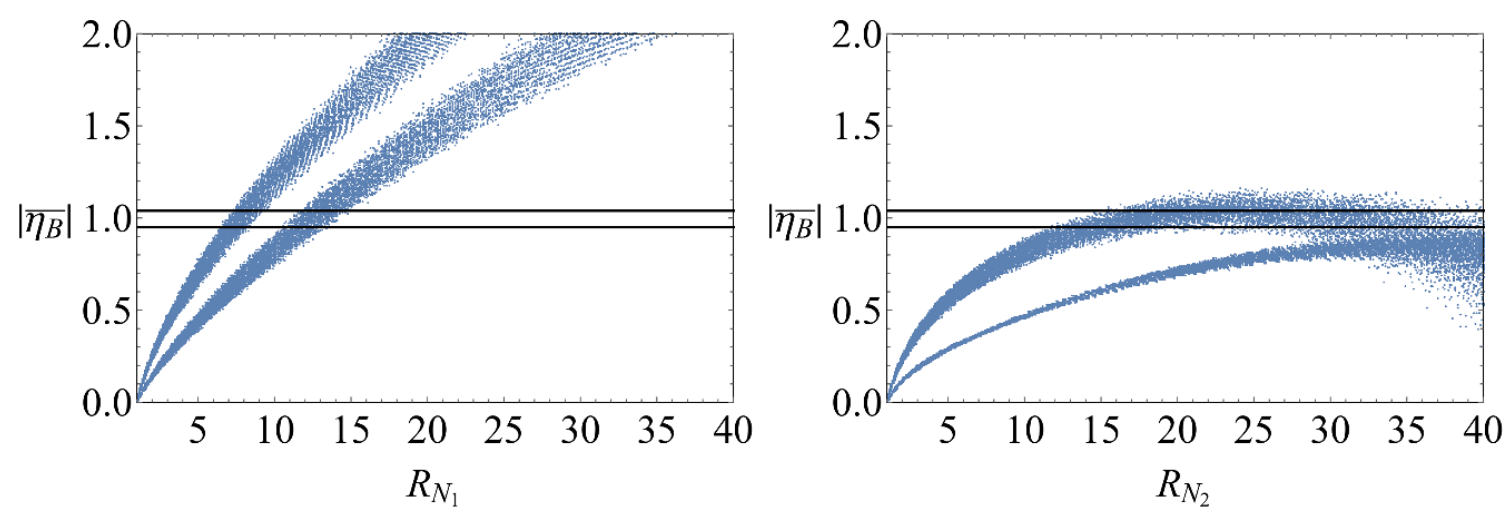

Figure 12. Normalised baryon to photon ratio with the $R$ parameters which quantify a particular $N_{i}$ dominance.

$M_{1}$ and hence, suppressed by the small values of $M_{1}$. Therefore, in order to produce the correct amount of CP violation, one must need the $N_{3}$ to couple with $N_{2}$.

Once $N_{3}$ is included in the discussion, we have more combinations of the $\mathrm{RH}$ mass spectrum on top of what has been shown in figure 5. However in this paper, we only consider the case $M_{3}>10^{12} \mathrm{GeV}$ so that the asymmetry generated by $M_{3}$ vanishes (due to $\mathrm{CP}^{\mu \tau}$ ) and we have contributions from $N_{2}$ with $10^{9} \mathrm{GeV}<M_{2}<10^{12} \mathrm{GeV}$. Note that this mass spectrum ${ }^{13}\left(M_{3} \gg M_{2} \gg M_{1}\right)$, implies a strong hierarchical scenario. Thus, unlike the case discussed in the earlier section, any of the components of the asymmetry generated by $N_{2}$ does not escape $N_{1}$-washout since, for this mass spectrum of the RH neutrinos, $M_{1}$ is in the $3 \mathrm{FR}$ and the directions of $N_{1}$-washout coincide with that of the charged leptons.

Following the above discussion, neglecting the contribution from $N_{1}$ and using eq. (4.23)-eq. (4.25), $B-L$ asymmetry parameter can now be written as

$$
N_{B-L}=-\varepsilon_{2 \tau} \kappa_{2 \tau}^{\infty} e^{-3 \pi K_{1 \tau} / 8}-\frac{K_{2 \mu}}{K_{2 \tau^{\perp}}} \varepsilon_{2 \tau^{\perp}} \kappa_{2 \tau^{\perp}}^{\infty} e^{-3 \pi K_{1 \mu} / 8}-\frac{K_{2 e}}{K_{2 \tau^{\perp}}} \varepsilon_{2 \tau^{\perp}} \kappa_{2 \tau^{\perp}}^{\infty} e^{-3 \pi K_{1 e} / 8} .
$$

Note that each term in the r.h.s. of eq. (5.18) contains the exponential washout factor involving the flavoured decay parameters. Thus strength of the $N_{1}$-decay parameters would finally decide whether the asymmetry generated by $N_{2}$ would survive against $N_{1}$-washout. Typically, $K_{1 \alpha}<1$ is the condition for the washout processes to be considered ineffective (see $[79,80]$ ), and thus $P\left(K_{1 \alpha}<1\right)$ is the probability for the asymmetry generated by $N_{2}$ to survive in the direction of ' $\alpha$ '. Given a general seesaw formula (constituent mass matrices are not subjected to any symmetry), it has been shown for hierarchical light neutrinos that $P\left(K_{1 e}<1\right): P\left(K_{1 \mu}<1\right): P\left(K_{1 \tau}<1\right) \simeq 0.36: 0.058: 0.067 \simeq 6.2: 1: 1.15$ [80]. For the $\mathrm{CP}^{\mu \tau}$ symmetric case, it is natural to infer that these probabilities would decrease, since in this case due to the imposed symmetry, there are now lesser number of parameters in the light neutrino mass matrix. For example., we compute these probabilities assuming

\footnotetext{
${ }^{13}$ This is a very interesting mass spectrum for which a particular RH neutrino lies in a particular flavour regime, i.e., $M_{3}$ is in $1 \mathrm{FR}, M_{2}$ is in $2 \mathrm{FR}$ and $M_{1}$ is in $3 \mathrm{FR}$. This mass spectrum is often realized in $\mathrm{SO}(10)$ models [77, 123, 124].
} 

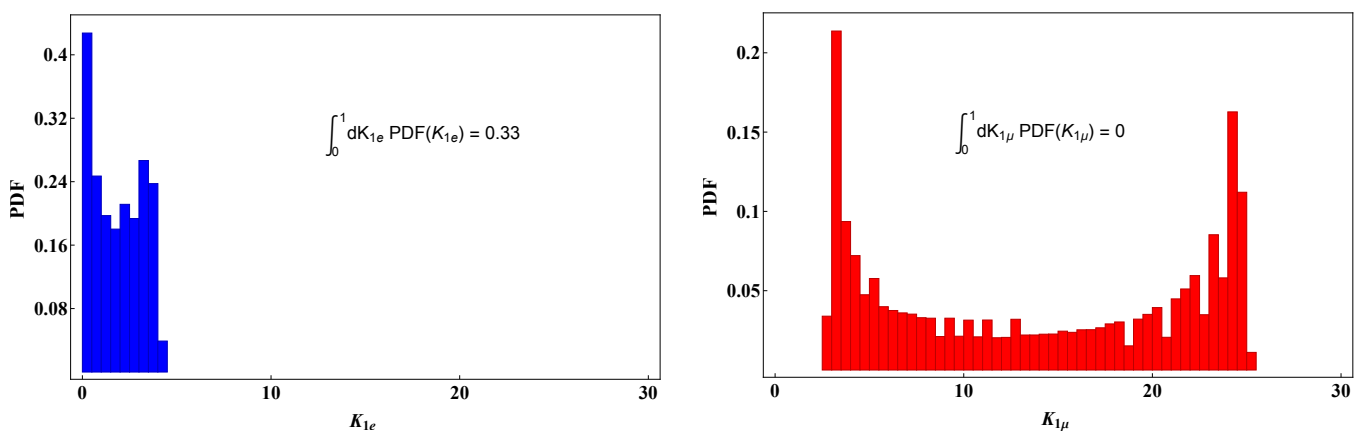

Figure 13. Distributions of the flavoured decay parameters. The probability for the electron decay parameter $K_{1 e}$ being less than 1 is almost $33 \%$ which corresponds to the fact that asymmetry generated by $N_{2}$ is most likely to survive against $N_{1}$ washout in the electron flavour.
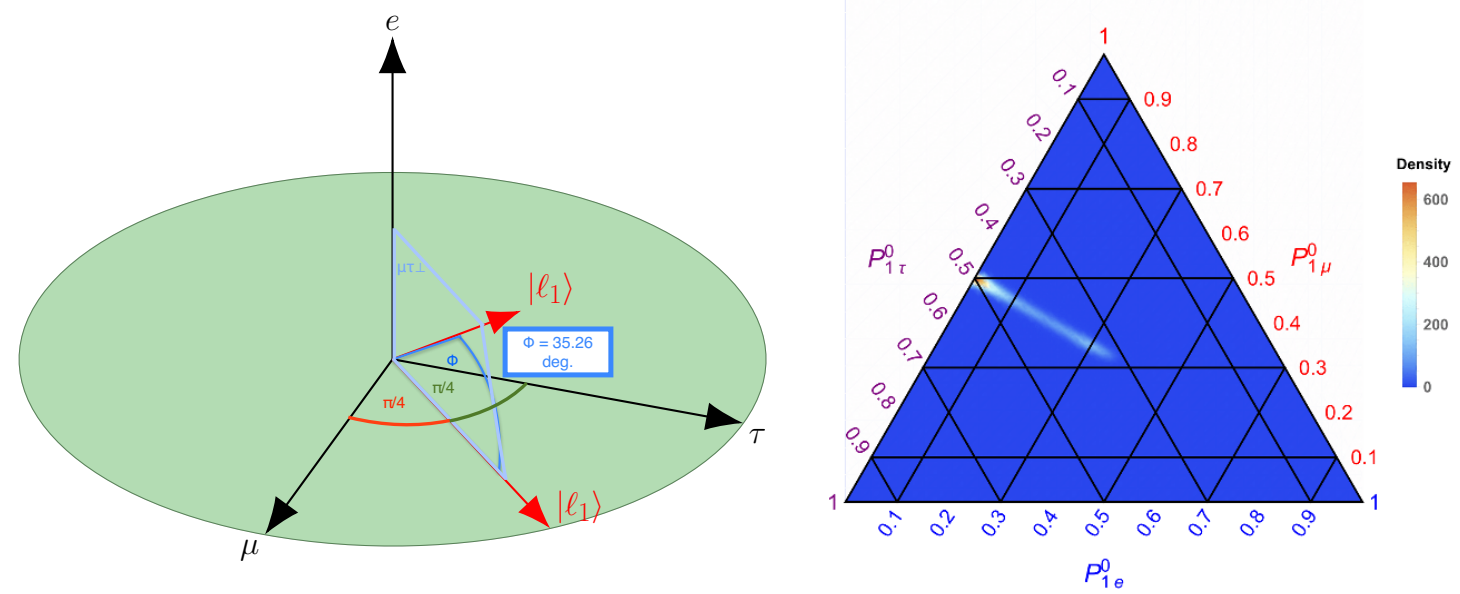

Figure 14. Left: possible range of the orientation of the state $|\ell\rangle_{1}$ in the CP symmetric model with hierarchical light neutrinos. Right: in the same model, ternary plot for the probabilities $P_{1 \alpha}=K_{1 \alpha} / \sum_{\alpha} K_{1 \alpha}$ with corresponding densities.

hierarchical light neutrinos ${ }^{14}$ and in figure 13 , we show the corresponding distributions. It is evident that, though for the electron flavour we have $P\left(K_{1 e}<1\right) \sim 0.33$, for the other two flavours (having same distribution due the $\mu \tau$ symmetry), the parameter space for $P\left(K_{1 \mu, \tau}<1\right)$ closes. This implies that the asymmetry generated by $N_{2}$ would survive in the electron flavour only. Note that since smaller values of $K_{1 e}$ are most probable, there are more number of points for the smaller values of $P_{1 e}=K_{1 e} / K_{1}$. This implies the states $|\ell\rangle_{1}$ tend to lie on the $\mu-\tau$ plane. The feature of getting mostly smaller values of $P_{1 e}$ is quite generic [80], but there is a clear difference between the general case and $\mathrm{CP}^{\mu \tau}$. For the latter, the entire $\mu-\tau$ plane is not accessible to $|\ell\rangle_{1}$, since in this case $P_{1 \mu}=P_{1 \tau}$, and therefore the state $|\ell\rangle_{1}$ will have a definite direction $\left(45^{0}\right.$ w.r.t. $\mu$ or $\tau$ axis $)$ on the $\mu-\tau$ plane. In addition, all possible orientations of the $|\ell\rangle_{1}$ will lie on the plane $\mu \tau \perp$ as shown in the left panel of figure 14. In the right panel, we show the triangle plot for the $P_{1 \alpha}$

\footnotetext{
${ }^{14}$ In our case assuming $N_{3}$ has Yukawa couplings $\left(m_{D}\right)_{3 \alpha}$ which are similar order of magnitude as that of $N_{1}$ or $N_{2}$ so that in the seesaw light neutrino mass matrix $\left(m_{D}\right)_{3 \alpha}$ is suppressed by the mass of $M_{3}$.
} 
with corresponding densities. It is evident that the maximum dense region corresponds to $P_{1 \mu}=P_{1 \tau}=0.5$ and the probability densities can have values upto the center of mass of the probability triangle, i.e., $P_{1 e}: P_{1 \mu}: P_{1 \tau} \simeq 1: 1: 1$. This suggests that there would be an upper limit (in this model $\sim 35.26^{0}$ ) on the angle $\Phi$ which measures the angular deviation of the state $|\ell\rangle_{1}$ from the $\mu \tau$ plane as shown in figure 14 .

\section{Summary}

In this work, we have performed a detailed study of the flavoured leptogenesis scenario in $\mathrm{CP}^{\mu \tau}$ symmetric neutrino mass models. We have shown how a mildly hierarchical leptogenesis $\left(M_{2} \simeq 4.7 M_{1}\right)$ can be realized within the two flavour regime. Within this class of models, even within the $N_{1}$-dominated scenario, the previously existing lower bound on $M_{1}$ can further be lowered approximately by an order of magnitude. Contrary to the previous works we have shown how in the two flavour regime, one can have a comparable parameter space for $N_{2}$ - leptogenesis in addition to the standard $N_{1}$ - leptogenesis. We have quantified the relevant mass scales of the RH neutrinos for a $N_{i}$-leptogenesis to dominate.

Taking the appropriate flavour effects into account, we have argued that the standard hierarchical $N_{1}$-dominated scenario is valid only for the mass window $\left(M_{1}^{\max }\right) \sim 4 \times 10^{10} \mathrm{GeV}$ $>M_{1}>\left(M_{1}^{\min }\right) \sim 7.5 \times 10^{9} \mathrm{GeV}$. Else, if the mass of $N_{1}$ goes beyond $M_{1}^{\max }$, there is a substantial amount of parameter space for which a $N_{2}$-dominated scenario could also be realized. We have considered other mass spectra of the heavy neutrinos for which the lepton asymmetry generated by $N_{2}$ in two flavour regime faces washout by $N_{1}$ in the three flavour regime. For a hierarchical light neutrino mass spectrum, we have demonstrated that approximately one third of the parameter space allows an electron-flavoured $N_{2}$-leptogenesis to be realized.

The possibility of having a mildly hierarchical leptogenesis opens up several interesting avenues. With this detailed work, we hope to elucidate some aspects of this involved problem. Certainly, inclusion of several other effects, e.g., consideration of flavour couplings, quantum corrections to the neutrino parameters would improve the results presented in this paper. We plan to include these effects in a future work.

\section{Acknowledgments}

We would like to thank Pasquale Di Bari for many useful discussions regarding leptogenesis. RS is supported by a Newton International Fellowship (NF 171202) from Royal Society (UK) and SERB (India). MS acknowledges support from the National Science Foundation, Grant PHY-1630782, and to the Heising-Simons Foundation, Grant 2017-228.

Open Access. This article is distributed under the terms of the Creative Commons Attribution License (CC-BY 4.0), which permits any use, distribution and reproduction in any medium, provided the original author(s) and source are credited. 


\section{References}

[1] Particle Data Group collaboration, Review of Particle Physics, Phys. Rev. D 98 (2018) 030001 [INSPIRE].

[2] T2K collaboration, Combined Analysis of Neutrino and Antineutrino Oscillations at T2K, Phys. Rev. Lett. 118 (2017) 151801 [arXiv:1701.00432] [INSPIRE].

[3] T2K collaboration, Measurement of neutrino and antineutrino oscillations by the T2K experiment including a new additional sample of $\nu_{e}$ interactions at the far detector, Phys. Rev. D 96 (2017) 092006 [Erratum ibid. D 98 (2018) 019902] [arXiv:1707.01048] [INSPIRE].

[4] T2K collaboration, Search for CP-violation in Neutrino and Antineutrino Oscillations by the T2K Experiment with $2.2 \times 10^{21}$ Protons on Target, Phys. Rev. Lett. 121 (2018) 171802 [arXiv: 1807.07891] [INSPIRE].

[5] NOvA collaboration, Constraints on Oscillation Parameters from $\nu_{e}$ Appearance and $\nu_{\mu}$ Disappearance in NOvA, Phys. Rev. Lett. 118 (2017) 231801 [arXiv:1703.03328] [INSPIRE].

[6] NOvA collaboration, New constraints on oscillation parameters from $\nu_{e}$ appearance and $\nu_{\mu}$ disappearance in the NOvA experiment, Phys. Rev. D 98 (2018) 032012 [arXiv: 1806.00096] [INSPIRE].

[7] I. Esteban, M.C. Gonzalez-Garcia, A. Hernandez-Cabezudo, M. Maltoni and T. Schwetz, Global analysis of three-flavour neutrino oscillations: synergies and tensions in the determination of $\theta_{23}, \delta_{C P}$ and the mass ordering, JHEP 01 (2019) 106 [arXiv: 1811.05487] [INSPIRE].

[8] G. Altarelli and F. Feruglio, Discrete Flavor Symmetries and Models of Neutrino Mixing, Rev. Mod. Phys. 82 (2010) 2701 [arXiv: 1002.0211] [InSPIRE].

[9] H. Ishimori, T. Kobayashi, H. Ohki, Y. Shimizu, H. Okada and M. Tanimoto, Non-Abelian Discrete Symmetries in Particle Physics, Prog. Theor. Phys. Suppl. 183 (2010) 1 [arXiv: 1003.3552] [INSPIRE].

[10] S.F. King, Unified Models of Neutrinos, Flavour and CP-violation, Prog. Part. Nucl. Phys. 94 (2017) 217 [arXiv: 1701.04413] [INSPIRE].

[11] S.T. Petcov, Discrete Flavour Symmetries, Neutrino Mixing and Leptonic CP-violation, Eur. Phys. J. C 78 (2018) 709 [arXiv:1711.10806] [INSPIRE].

[12] R.N. Mohapatra and S. Nussinov, Bimaximal neutrino mixing and neutrino mass matrix, Phys. Rev. D 60 (1999) 013002 [hep-ph/9809415] [INSPIRE].

[13] C.S. Lam, A 2-3 symmetry in neutrino oscillations, Phys. Lett. B 507 (2001) 214 [hep-ph/0104116] [INSPIRE].

[14] E. Ma and M. Raidal, Neutrino mass, muon anomalous magnetic moment and lepton flavor nonconservation, Phys. Rev. Lett. 87 (2001) 011802 [Erratum ibid. 87 (2001) 159901] [hep-ph/0102255] [INSPIRE].

[15] K.R.S. Balaji, W. Grimus and T. Schwetz, The Solar LMA neutrino oscillation solution in the Zee model, Phys. Lett. B 508 (2001) 301 [hep-ph/0104035] [INSPIRE].

[16] T. Fukuyama and H. Nishiura, Mass matrix of Majorana neutrinos, hep-ph/9702253 [INSPIRE]. 
[17] T. Fukuyama, Twenty years after the discovery of $\mu-\tau$ symmetry, PTEP 2017 (2017) 033B11 [arXiv: 1701.04985] [INSPIRE].

[18] R.N. Mohapatra and W. Rodejohann, Broken mu-tau symmetry and leptonic CP-violation, Phys. Rev. D 72 (2005) 053001 [hep-ph/0507312] [INSPIRE].

[19] Z.-z. Xing, H. Zhang and S. Zhou, Nearly Tri-bimaximal Neutrino Mixing and CP-violation from mu-tau Symmetry Breaking, Phys. Lett. B 641 (2006) 189 [hep-ph/0607091] [INSPIRE].

[20] P.F. Harrison and W.G. Scott, $\mu-\tau$ reflection symmetry in lepton mixing and neutrino oscillations, Phys. Lett. B 547 (2002) 219 [hep-ph/0210197] [INSPIRE].

[21] W. Grimus and L. Lavoura, A Nonstandard CP transformation leading to maximal atmospheric neutrino mixing, Phys. Lett. B 579 (2004) 113 [hep-ph/0305309] [INSPIRE].

[22] R.N. Mohapatra and C.C. Nishi, Implications of $\mu-\tau$ flavored CP symmetry of leptons, JHEP 08 (2015) 092 [arXiv:1506.06788] [INSPIRE].

[23] R.N. Mohapatra and C.C. Nishi, $S_{4}$ Flavored CP Symmetry for Neutrinos, Phys. Rev. D 86 (2012) 073007 [arXiv: 1208.2875] [INSPIRE].

[24] S. Gupta, A.S. Joshipura and K.M. Patel, Minimal extension of tri-bimaximal mixing and generalized $Z_{2} \times Z_{2}$ symmetries, Phys. Rev. D 85 (2012) 031903 [arXiv:1112.6113] [INSPIRE].

[25] F. Feruglio, C. Hagedorn and R. Ziegler, Lepton Mixing Parameters from Discrete and CP Symmetries, JHEP 07 (2013) 027 [arXiv:1211.5560] [INSPIRE].

[26] M. Holthausen, M. Lindner and M.A. Schmidt, CP and Discrete Flavour Symmetries, JHEP 04 (2013) 122 [arXiv:1211.6953] [INSPIRE].

[27] M.-C. Chen, M. Fallbacher, K.T. Mahanthappa, M. Ratz and A. Trautner, CP Violation from Finite Groups, Nucl. Phys. B 883 (2014) 267 [arXiv:1402.0507] [InSPIRE].

[28] G.-J. Ding, S.F. King, C. Luhn and A.J. Stuart, Spontaneous CP-violation from vacuum alignment in $S_{4}$ models of leptons, JHEP 05 (2013) 084 [arXiv:1303.6180] [INSPIRE].

[29] F. Feruglio, C. Hagedorn and R. Ziegler, A realistic pattern of lepton mixing and masses from $S_{4}$ and $C P$, Eur. Phys. J. C $\mathbf{7 4}$ (2014) 2753 [arXiv:1303.7178] [InSPIRE].

[30] C.C. Nishi and B.L. Sánchez-Vega, Mu-tau reflection symmetry with a texture-zero, JHEP 01 (2017) 068 [arXiv:1611.08282] [INSPIRE].

[31] W. Rodejohann and X.-J. Xu, Trimaximal $\mu-\tau$ reflection symmetry, Phys. Rev. D 96 (2017) 055039 [arXiv: 1705. 02027] [INSPIRE].

[32] J.T. Penedo, S.T. Petcov and A.V. Titov, Neutrino mixing and leptonic CP-violation from $S_{4}$ flavour and generalised CP symmetries, JHEP 12 (2017) 022 [arXiv:1705.00309] [INSPIRE].

[33] R. Samanta and A. Ghosal, Probing maximal zero textures with broken cyclic symmetry in inverse seesaw, Nucl. Phys. B 911 (2016) 846 [arXiv:1507.02582] [INSPIRE].

[34] Z.-h. Zhao, Modifications to the neutrino mixing from the $\mu-\tau$ reflection symmetry, Nucl. Phys. B 935 (2018) 129 [arXiv: 1803.04603] [InSPIRE].

[35] R. Samanta, P. Roy and A. Ghosal, Consequences of minimal seesaw with complex $\mu \tau$ antisymmetry of neutrinos, JHEP 06 (2018) 085 [arXiv: 1712.06555] [INSPIRE]. 
[36] S.F. King and C.C. Nishi, Mu-tau symmetry and the Littlest Seesaw, Phys. Lett. B 785 (2018) 391 [arXiv: 1807.00023] [INSPIRE].

[37] Z.-C. Liu, C.-X. Yue and Z.-H. Zhao, On the textures of neutrino mass matrix for maximal atmospheric mixing angle and Dirac CP phase, JHEP 10 (2018) 106 [arXiv:1807.10031] [INSPIRE].

[38] M.H. Rahat, P. Ramond and B. Xu, Asymmetric tribimaximal texture, Phys. Rev. D 98 (2018) 055030 [arXiv: 1805.10684] [INSPIRE].

[39] C.C. Nishi, B.L. Sánchez-Vega and G. Souza Silva, $\mu \tau$ reflection symmetry with a high scale texture-zero, JHEP 09 (2018) 042 [arXiv: 1806.07412] [INSPIRE].

[40] D.M. Barreiros, R.G. Felipe and F.R. Joaquim, Combining texture zeros with a remnant CP symmetry in the minimal type-I seesaw, JHEP 01 (2019) 223 [arXiv:1810.05454] [INSPIRE].

[41] F.J. de Anda, S.F. King and E. Perdomo, SU(5) Grand Unified Theory with $A_{4}$ Modular Symmetry, arXiv:1812.05620 [INSPIRE].

[42] S.F. King and Y.-L. Zhou, Littlest mu-tau seesaw, JHEP 05 (2019) 217 [arXiv: 1901.06877] [INSPIRE].

[43] G. Ecker, W. Grimus and H. Neufeld, A Standard Form for Generalized CP Transformations, J. Phys. A 20 (1987) L807 [InSPIRE].

[44] W. Grimus and M.N. Rebelo, Automorphisms in gauge theories and the definition of $C P$ and P, Phys. Rept. 281 (1997) 239 [hep-ph/9506272] [INSPIRE].

[45] P. Chen, C.-Y. Yao and G.-J. Ding, Neutrino Mixing from CP Symmetry, Phys. Rev. D 92 (2015) 073002 [arXiv: 1507.03419] [inSPIRE].

[46] E. Ma, Neutrino mixing: $A_{4}$ variations, Phys. Lett. B 752 (2016) 198 [arXiv:1510.02501] [INSPIRE].

[47] P. Chen, G.-J. Ding, F. Gonzalez-Canales and J.W.F. Valle, Generalized $\mu-\tau$ reflection symmetry and leptonic CP-violation, Phys. Lett. B 753 (2016) 644 [arXiv:1512.01551] [INSPIRE].

[48] P. Chen, G.-J. Ding, F. Gonzalez-Canales and J.W.F. Valle, Classifying CP transformations according to their texture zeros: theory and implications, Phys. Rev. D 94 (2016) 033002 [arXiv: 1604.03510] [INSPIRE].

[49] R. Sinha, P. Roy and A. Ghosal, CP transformed mixed $\mu \tau$ antisymmetry for neutrinos and its consequences, Phys. Rev. D 99 (2019) 033009 [arXiv: 1809.06615] [INSPIRE].

[50] R. Samanta, P. Roy and A. Ghosal, Extended scaling and residual flavor symmetry in the neutrino Majorana mass matrix, Eur. Phys. J. C 76 (2016) 662 [arXiv:1604.06731] [INSPIRE].

[51] R. Samanta, P. Roy and A. Ghosal, Complex Scaling in Neutrino Mass Matrix, Acta Phys. Polon. Supp. 9 (2016) 807 [arXiv:1604.01206] [INSPIRE].

[52] R. Samanta, M. Chakraborty, P. Roy and A. Ghosal, Baryon asymmetry via leptogenesis in a neutrino mass model with complex scaling, JCAP 03 (2017) 025 [arXiv:1610.10081] [INSPIRE].

[53] A. Ghosal and R. Samanta, Probing texture zeros with scaling ansatz in inverse seesaw, JHEP 05 (2015) 077 [arXiv:1501.00916] [INSPIRE]. 
[54] R. Samanta and M. Chakraborty, A study on a minimally broken residual TBM-Klein symmetry with its implications on flavoured leptogenesis and ultra high energy neutrino flux ratios, JCAP 02 (2019) 003 [arXiv: 1802.04751] [INSPIRE].

[55] R. Sinha, R. Samanta and A. Ghosal, Generalized $\mathbb{Z}_{2} \times \mathbb{Z}_{2}$ in scaling neutrino Majorana mass matrix and baryogenesis via flavored leptogenesis, JHEP 12 (2017) 030 [arXiv: 1706.00946] [INSPIRE].

[56] R. Sinha, S. Bhattacharya and R. Samanta, Phenomenological implications of the Friedberg-Lee transformation in a neutrino mass model with $\mu \tau$-flavored CP symmetry, JHEP 03 (2019) 081 [arXiv:1810.05391] [INSPIRE].

[57] V.A. Kuzmin, V.A. Rubakov and M.E. Shaposhnikov, On the Anomalous Electroweak Baryon Number Nonconservation in the Early Universe, Phys. Lett. 155B (1985) 36 [INSPIRE].

[58] M. Fukugita and T. Yanagida, Baryogenesis Without Grand Unification, Phys. Lett. B 174 (1986) 45 [inSPIRE].

[59] A. Riotto and M. Trodden, Recent progress in baryogenesis, Ann. Rev. Nucl. Part. Sci. 49 (1999) 35 [hep-ph/9901362] [inSPIRE].

[60] E.W. Kolb and M.S. Turner, The Early Universe, Front. Phys. 69 (1990) 1 [InSPIRE].

[61] A. Pilaftsis and T.E.J. Underwood, Resonant leptogenesis, Nucl. Phys. B 692 (2004) 303 [hep-ph/0309342] [INSPIRE].

[62] F.F. Deppisch and A. Pilaftsis, Lepton Flavour Violation and $\theta_{13}$ in Minimal Resonant Leptogenesis, Phys. Rev. D 83 (2011) 076007 [arXiv:1012.1834] [INSPIRE].

[63] W. Buchmüller, P. Di Bari and M. Plümacher, Leptogenesis for pedestrians, Annals Phys. 315 (2005) 305 [hep-ph/0401240] [INSPIRE].

[64] S. Davidson, E. Nardi and Y. Nir, Leptogenesis, Phys. Rept. 466 (2008) 105 [arXiv:0802.2962] [INSPIRE].

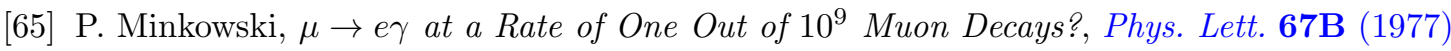
421 [INSPIRE].

[66] M. Gell-Mann, P. Ramond and R. Slansky, Complex Spinors and Unified Theories, Conf. Proc. C 790927 (1979) 315 [arXiv: 1306.4669] [INSPIRE].

[67] R.N. Mohapatra and G. Senjanović, Neutrino Mass and Spontaneous Parity Nonconservation, Phys. Rev. Lett. 44 (1980) 912 [INSPIRE].

[68] PLANCK collaboration, Planck intermediate results. XLVI. Reduction of large-scale systematic effects in HFI polarization maps and estimation of the reionization optical depth, Astron. Astrophys. 596 (2016) A107 [arXiv:1605. 02985] [INSPIRE].

[69] P. Chen, G.-J. Ding and S.F. King, Leptogenesis and residual CP symmetry, JHEP 03 (2016) 206 [arXiv:1602.03873] [INSPIRE].

[70] C. Hagedorn and E. Molinaro, Flavor and CP symmetries for leptogenesis and $0 \nu \beta \beta$ decay, Nucl. Phys. B 919 (2017) 404 [arXiv:1602.04206] [inSPIRE].

[71] A. Abada, S. Davidson, A. Ibarra, F.X. Josse-Michaux, M. Losada and A. Riotto, Flavour Matters in Leptogenesis, JHEP 09 (2006) 010 [hep-ph/0605281] [INSPIRE].

[72] E. Nardi, Y. Nir, E. Roulet and J. Racker, The Importance of flavor in leptogenesis, JHEP 01 (2006) 164 [hep-ph/0601084] [INSPIRE]. 
[73] S. Blanchet and P. Di Bari, Flavor effects on leptogenesis predictions, JCAP 03 (2007) 018 [hep-ph/0607330] [INSPIRE].

[74] P.S.B. Dev, P. Di Bari, B. Garbrecht, S. Lavignac, P. Millington and D. Teresi, Flavor effects in leptogenesis, Int. J. Mod. Phys. A 33 (2018) 1842001 [arXiv:1711.02861] [INSPIRE].

[75] S.F. King, Large mixing angle $M S W$ and atmospheric neutrinos from single right-handed neutrino dominance and U(1) family symmetry, Nucl. Phys. B 576 (2000) 85 [hep-ph/9912492] [INSPIRE].

[76] S.F. King, Constructing the large mixing angle MNS matrix in seesaw models with right-handed neutrino dominance, JHEP 09 (2002) 011 [hep-ph/0204360] [INSPIRE].

[77] P. Di Bari and A. Riotto, Successful type-I Leptogenesis with $\mathrm{SO}(10)$-inspired mass relations, Phys. Lett. B 671 (2009) 462 [arXiv:0809.2285] [INSPIRE].

[78] S. Blanchet, P. Di Bari, D.A. Jones and L. Marzola, Leptogenesis with heavy neutrino flavours: from density matrix to Boltzmann equations, JCAP 01 (2013) 041 [arXiv:1112.4528] [INSPIRE].

[79] P. Di Bari, Seesaw geometry and leptogenesis, Nucl. Phys. B 727 (2005) 318 [hep-ph/0502082] [INSPIRE].

[80] P. Di Bari, M. Re Fiorentin and R. Samanta, Representing seesaw neutrino models and their motion in lepton flavour space, JHEP 05 (2019) 011 [arXiv: 1812.07720] [INSPIRE].

[81] P.H. Frampton, S.L. Glashow and T. Yanagida, Cosmological sign of neutrino CP-violation, Phys. Lett. B 548 (2002) 119 [hep-ph/0208157] [INSPIRE].

[82] M. Raidal and A. Strumia, Predictions of the most minimal seesaw model, Phys. Lett. B 553 (2003) 72 [hep-ph/0210021] [INSPIRE].

[83] S. Antusch, P. Di Bari, D.A. Jones and S.F. King, Leptogenesis in the Two Right-Handed Neutrino Model Revisited, Phys. Rev. D 86 (2012) 023516 [arXiv:1107.6002] [INSPIRE].

[84] G. Bambhaniya, P.S. Bhupal Dev, S. Goswami, S. Khan and W. Rodejohann, Naturalness, Vacuum Stability and Leptogenesis in the Minimal Seesaw Model, Phys. Rev. D 95 (2017) 095016 [arXiv: 1611.03827] [INSPIRE].

[85] W. Grimus, S. Kaneko, L. Lavoura, H. Sawanaka and M. Tanimoto, mu-tau antisymmetry and neutrino mass matrices, JHEP 01 (2006) 110 [hep-ph/0510326] [INSPIRE].

[86] G.F. Giudice, M. Peloso, A. Riotto and I. Tkachev, Production of massive fermions at preheating and leptogenesis, JHEP 08 (1999) 014 [hep-ph/9905242] [INSPIRE].

[87] M.Yu. Khlopov and A.D. Linde, Is It Easy to Save the Gravitino?, Phys. Lett. 138B (1984) 265 [INSPIRE].

[88] G.F. Giudice, A. Notari, M. Raidal, A. Riotto and A. Strumia, Towards a complete theory of thermal leptogenesis in the SM and MSSM, Nucl. Phys. B 685 (2004) 89 [hep-ph/0310123] [INSPIRE].

[89] D. Croon, N. Fernandez, D. McKeen and G. White, Stability, reheating and leptogenesis, JHEP 06 (2019) 098 [arXiv: 1903.08658] [INSPIRE].

[90] W. Buchmüller, P. Di Bari and M. Plümacher, The Neutrino mass window for baryogenesis, Nucl. Phys. B 665 (2003) 445 [hep-ph/0302092] [INSPIRE]. 
[91] W. Buchmüller, P. Di Bari and M. Plümacher, Cosmic microwave background, matter-antimatter asymmetry and neutrino masses, Nucl. Phys. B 643 (2002) 367 [Erratum ibid. B 793 (2008) 362] [hep-ph/0205349] [INSPIRE].

[92] S. H.-S. Alexander, M.E. Peskin and M.M. Sheikh-Jabbari, Leptogenesis from gravity waves in models of inflation, Phys. Rev. Lett. 96 (2006) 081301 [hep-th/0403069] [InSPIRE].

[93] P. Adshead, A.J. Long and E.I. Sfakianakis, Gravitational Leptogenesis, Reheating and Models of Neutrino Mass, Phys. Rev. D 97 (2018) 043511 [arXiv:1711.04800] [INSPIRE].

[94] E. Bertuzzo, P. Di Bari and L. Marzola, The problem of the initial conditions in flavoured leptogenesis and the tauon $N_{2}$-dominated scenario, Nucl. Phys. B 849 (2011) 521 [arXiv: 1007.1641] [INSPIRE].

[95] G. Engelhard, Y. Grossman, E. Nardi and Y. Nir, The Importance of N2 leptogenesis, Phys. Rev. Lett. 99 (2007) 081802 [hep-ph/0612187] [INSPIRE].

[96] P. Di Bari and L. Marzola, $\mathrm{SO}(10)$-inspired solution to the problem of the initial conditions in leptogenesis, Nucl. Phys. B 877 (2013) 719 [arXiv:1308.1107] [INSPIRE].

[97] M. Chianese and P. Di Bari, Strong thermal SO(10)-inspired leptogenesis in the light of recent results from long-baseline neutrino experiments, JHEP 05 (2018) 073 [arXiv: 1802.07690] [INSPIRE].

[98] S. Blanchet and P. Di Bari, Leptogenesis beyond the limit of hierarchical heavy neutrino masses, JCAP 06 (2006) 023 [hep-ph/0603107] [INSPIRE].

[99] D. Aristizabal Sierra, L.A. Muñoz and E. Nardi, Purely Flavored Leptogenesis, Phys. Rev. D 80 (2009) 016007 [arXiv:0904.3043] [INSPIRE].

[100] S. Davidson and A. Ibarra, A Lower bound on the right-handed neutrino mass from leptogenesis, Phys. Lett. B 535 (2002) 25 [hep-ph/0202239] [INSPIRE].

[101] B. Adhikary, M. Chakraborty and A. Ghosal, Masses, mixing angles and phases of general Majorana neutrino mass matrix, JHEP 10 (2013) 043 [Erratum ibid. 09 (2014) 180] [arXiv:1307.0988] [INSPIRE].

[102] M. Agostini et al., Background-free search for neutrinoless double- $\beta$ decay of ${ }^{76}$ Ge with GERDA, arXiv:1703.00570 [INSPIRE].

[103] KamLAND-Zen collaboration, Limit on Neutrinoless $\beta \beta$ Decay of ${ }^{136}$ Xe from the First Phase of KamLAND-Zen and Comparison with the Positive Claim in ${ }^{76} \mathrm{Ge}$, Phys. Rev. Lett. 110 (2013) 062502 [arXiv:1211.3863] [INSPIRE].

[104] EXO-200 collaboration, Search for Neutrinoless Double-Beta Decay in ${ }^{136}$ Xe with EXO-200, Phys. Rev. Lett. 109 (2012) 032505 [arXiv:1205.5608] [INSPIRE].

[105] M. Agostini, G. Benato and J. Detwiler, Discovery probability of next-generation neutrinoless double- $\beta$ decay experiments, Phys. Rev. D 96 (2017) 053001 [arXiv: 1705. 02996] [INSPIRE].

[106] J. Shirai, Results and future plans for the KamLAND-Zen experiment, J. Phys. Conf. Ser. 888 (2017) 012031 [INSPIRE].

[107] B. Mong, nEXO Double Beta Decay, in HQL2016, Blacksburg, VA, U.S.A. (2016) [http://indico.phys.vt.edu/event/31/session/29/contribution/103/material/slides/0.pdf].

[108] CUPID collaboration, CUPID: CUORE (Cryogenic Underground Observatory for Rare Events) Upgrade with Particle IDentification, arXiv:1504.03599 [INSPIRE]. 
[109] CUORE collaboration, CUORE: A Cryogenic underground observatory for rare events, Nucl. Instrum. Meth. A 518 (2004) 775 [hep-ex/0212053] [INSPIRE].

[110] B. Schwingenheuer, Neutrinoless double beta decay with 76Ge, in CERN EP Seminar, Geneva, Switzerland (2017) [https://indico.cern.ch/event/581028/attachments/1395349/2129744/ge76_cern.pdf].

[111] R. Samanta, R. Sinha and A. Ghosal, Importance of generalized $\mu \tau$ symmetry and its $C P$ extension on neutrino mixing and leptogenesis, JHEP 10 (2019) 057 [arXiv:1805.10031] [INSPIRE].

[112] R. Barbieri, P. Creminelli, A. Strumia and N. Tetradis, Baryogenesis through leptogenesis, Nucl. Phys. B 575 (2000) 61 [hep-ph/9911315] [InSPIRE].

[113] S. Antusch, P. Di Bari, D.A. Jones and S.F. King, A fuller flavour treatment of $\mathrm{N}_{2}$-dominated leptogenesis, Nucl. Phys. B 856 (2012) 180 [arXiv:1003.5132] [InSPIRE].

[114] K. Moffat, S. Pascoli, S.T. Petcov, H. Schulz and J. Turner, Three-flavored nonresonant leptogenesis at intermediate scales, Phys. Rev. D 98 (2018) 015036 [arXiv:1804.05066] [INSPIRE].

[115] P.S. Bhupal Dev, P. Millington, A. Pilaftsis and D. Teresi, Flavour Covariant Transport Equations: an Application to Resonant Leptogenesis, Nucl. Phys. B 886 (2014) 569 [arXiv: 1404.1003] [INSPIRE].

[116] A. De Simone and A. Riotto, Quantum Boltzmann Equations and Leptogenesis, JCAP 08 (2007) 002 [hep-ph/0703175] [INSPIRE].

[117] M. Garny, A. Kartavtsev and A. Hohenegger, Leptogenesis from first principles in the resonant regime, Annals Phys. 328 (2013) 26 [arXiv:1112.6428] [INSPIRE].

[118] P.S. Bhupal Dev, P. Millington, A. Pilaftsis and D. Teresi, Kadanoff-Baym approach to flavour mixing and oscillations in resonant leptogenesis, Nucl. Phys. B 891 (2015) 128 [arXiv: 1410.6434] [INSPIRE].

[119] A. Kartavtsev, P. Millington and H. Vogel, Lepton asymmetry from mixing and oscillations, JHEP 06 (2016) 066 [arXiv:1601.03086] [INSPIRE].

[120] M.A. Luty, Baryogenesis via leptogenesis, Phys. Rev. D 45 (1992) 455 [inSPIRE].

[121] F. Hahn-Woernle, M. Plümacher and Y.Y.Y. Wong, Full Boltzmann equations for leptogenesis including scattering, JCAP 08 (2009) 028 [arXiv:0907.0205] [INSPIRE].

[122] M. Plümacher, Baryon asymmetry, neutrino mixing and supersymmetric $\mathrm{SO}(10)$ unification, Nucl. Phys. B 530 (1998) 207 [hep-ph/9704231] [INSPIRE].

[123] E.K. Akhmedov, M. Frigerio and A.Yu. Smirnov, Probing the seesaw mechanism with neutrino data and leptogenesis, JHEP 09 (2003) 021 [hep-ph/0305322] [INSPIRE].

[124] C.S. Fong, D. Meloni, A. Meroni and E. Nardi, Leptogenesis in SO(10), JHEP 01 (2015) 111 [arXiv:1412.4776] [INSPIRE]. 